\title{
The quenched SU(2) fundamental scalar propagator in minimal Landau gauge
}

\author{
Axel Maas ${ }^{\mathrm{a}}$ \\ Institute of Physics, NAWI Graz, University of Graz, Universitätsplatz 5, 8010 Graz, Austria
}

Received: 7 April 2016 / Accepted: 17 June 2016 / Published online: 2 July 2016

(C) The Author(s) 2016. This article is published with open access at Springerlink.com

\begin{abstract}
It is a long-standing question whether the confinement of matter fields in QCD has an imprint in the (gaugedependent) correlation functions, especially the propagators. As the analytic structure plays an important role in this question, high-precision data is necessary for lattice investigations. Also, it is interesting how this depends on the dimensionality of the theory. To make a study over a wide range of parameters possible this suggests to use scalar particles. This is done here: The propagator of a fundamental scalar is studied in two, three, and four dimensions in quenched SU(2) Yang-Mills theory in minimal Landau gauge, both in momentum space and position space. Particular emphasis is put on the effects of renormalization. The results suggest a quite intricate volume dependence and the presence of an intrinsic mass scale, but no obvious connection to confinement.
\end{abstract}

\section{Introduction}

The confinement of matter particles in QCD is a very longstanding problem [1]. In particular, the question is whether the properties of the propagator describing the elementary particles, both gluons and matter, show signs of confinement ${ }^{1}$ [2-8]. Of course, such investigations require one to fix a gauge to discuss the corresponding propagator, which will be here chosen to be the best-studied case so far, the Landau gauge, especially the so-called minimal Landau gauge [3].

One of the major tools used for this purpose is the spectral density. This spectral density is found to be positivity violating for gluons [2-10], though the precise form this violation takes, e.g. by a non-trivial cut structure, complex poles, or otherwise, is still under debate. At any rate, any violation of positivity immediately implies that the particle cannot be part of the physical state space, and thus not observable. Sufficient, but not necessary, conditions for violation of positivity

\footnotetext{
a e-mail: axelmaas@web.de
}

can be either a non-positive definite position-space correlation function or a non-monotonous behavior of the derivatives of the momentum-space correlation functions [3]. The form of such violations can then be used to constrain the type of analytic structure. For example, an oscillatory behavior in position space points to a complex pole structure [3].

The quark case is more intricate, see $[2,8,11-13]$, but there are also strong indications for a violation of positivity.

For scalar matter, so far little is known, though their much simpler Lorentz structure suggests them as a testbed. There have been investigations using functional methods which provide two possible scenarios for the behavior of the propagator, either being conventionally massive or possibly showing some quasi-conformal behavior [14-19].

There is also another intriguing question. In two (Euclidean) dimensions, i.e. one space and one time dimension, where gluons are not dynamical degrees of freedom, the violation of positivity for gluons appears to occur in much the same way as in higher dimensions [10,20-22]. However, in two dimensions gluons are not physical particles, but merely gauge degrees of freedom. At the same time, confinement according to the Wilson potential occurs already for purely geometrical reasons [23]. Scalar particles are, however, also in two dimensions physical particles. Whether their analytical structure changes may therefore give a hint as to how geometrical and dynamical confinement differ. Of course, this requires one to study the propagator in the quenched case, where the Wilson potential is indefinitely rising also in higher dimensions. Fermions, on the other hand, are quite differently affected by changing the dimensionality, thus intertwining many different aspects.

\footnotetext{
${ }^{1}$ Confinement is here understood, if not stated otherwise, in the sense that a particle cannot be observed as an asymptotic, physical state. In this sense also QCD is confining. A definition of confinement based on the Wilson string tension is in no obvious way related to this. In fact, with this view on confinement QCD is not a confining theory. See [1] for a more detailed discussion of this difference.
} 

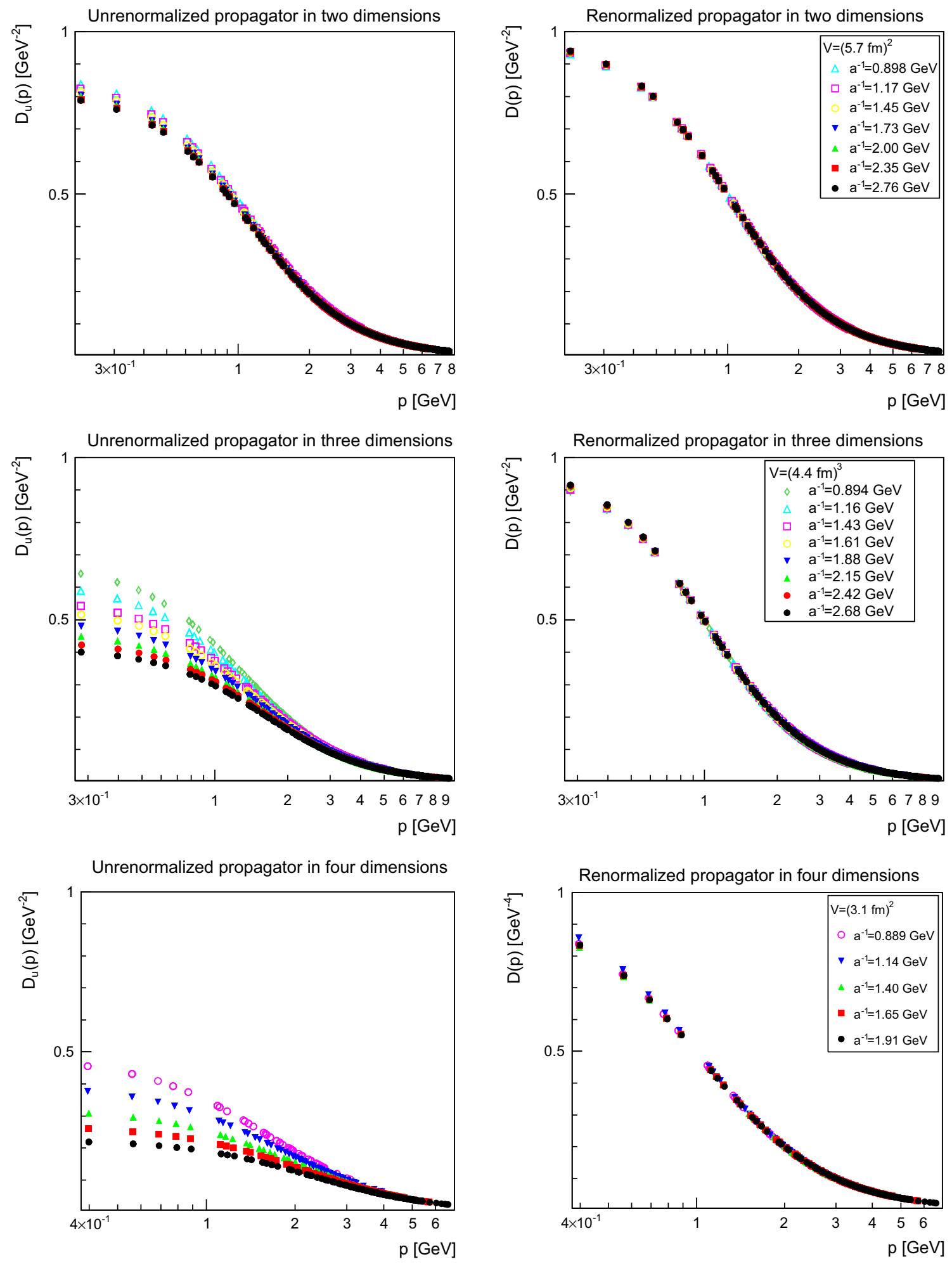

Fig. 1 Unrenormalized propagator (left panels) and renormalized propagator (right panels). The top panels are for two dimensions, the middle panels for three dimensions, and the bottom panels for four

dimensions. The values are $m_{\mathrm{r}}=m=1 \mathrm{GeV}$ and $\mu=1.5 \mathrm{GeV}$. If the (statistical $1 \sigma$ ) error bars here and hereafter are not visible then they are smaller than the symbol size 

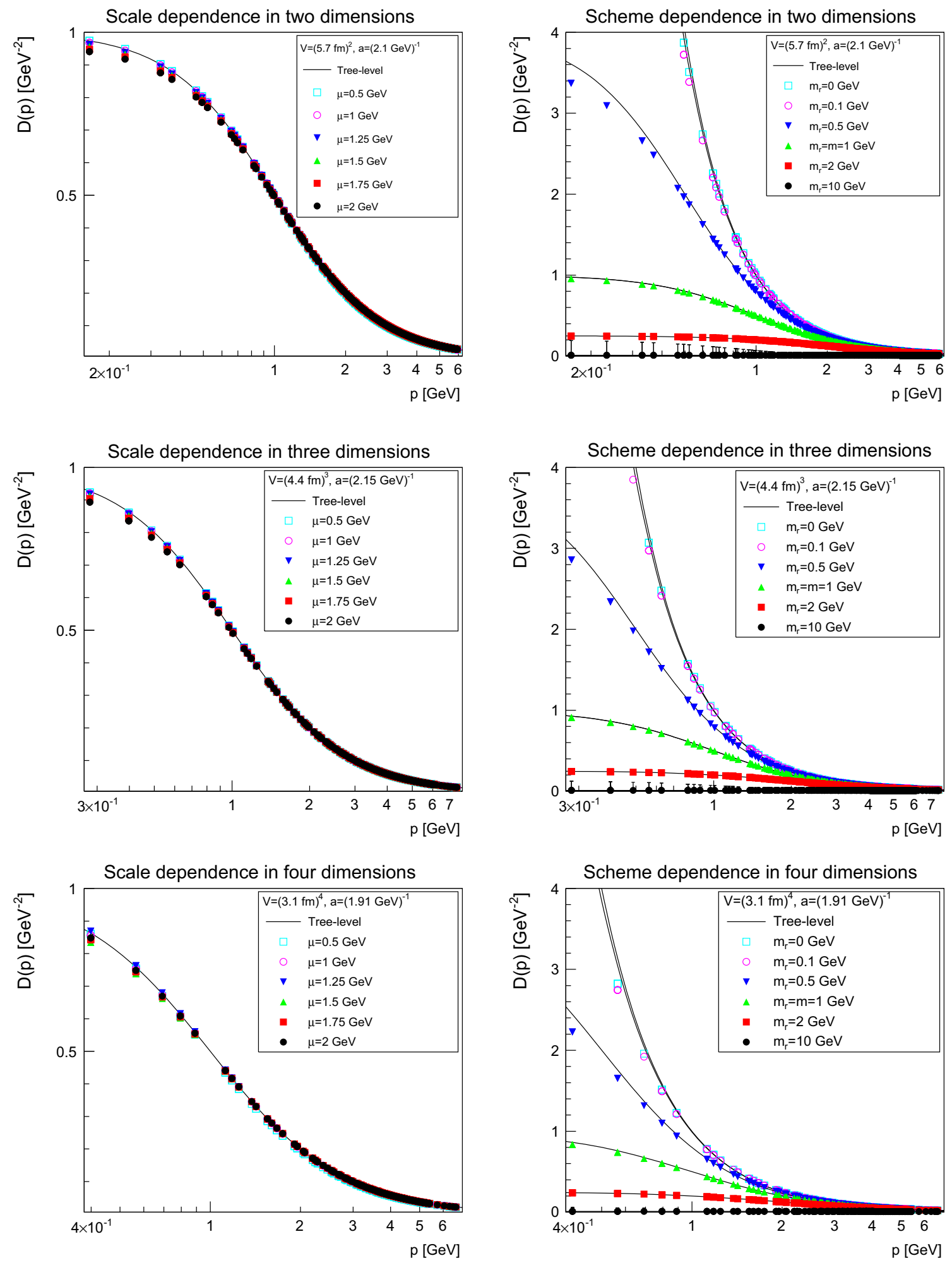

Fig. 2 Scale dependence at $m_{\mathrm{r}}=m=1 \mathrm{GeV}$ (left panels) and scheme dependence at $\mu=1.5 \mathrm{GeV}$ (right panels) of the renormalized propagator. The top panels are two dimensions, the middle panels three dimen-

sions, and the bottom panels four dimensions. The tree-level propagator is shown for comparison as a full line 
Wave-function renormalization constant in two dimensions

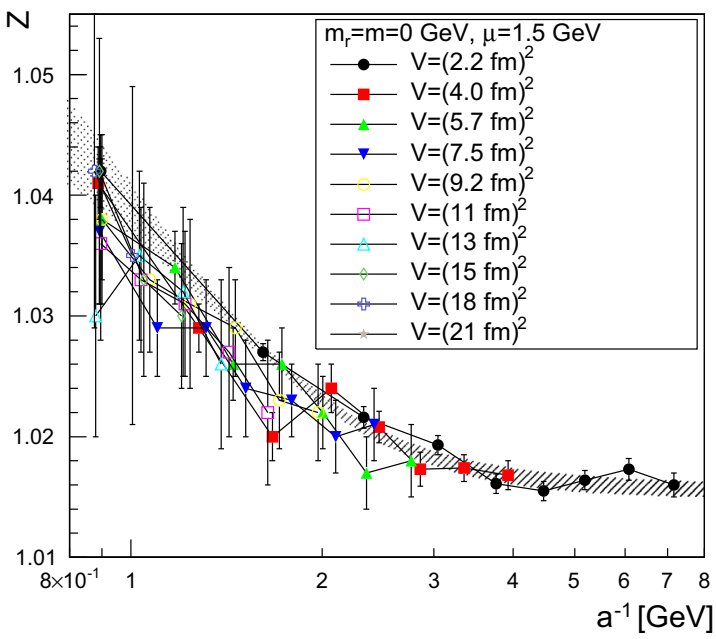

Wave-function renormalization constant in two dimensions

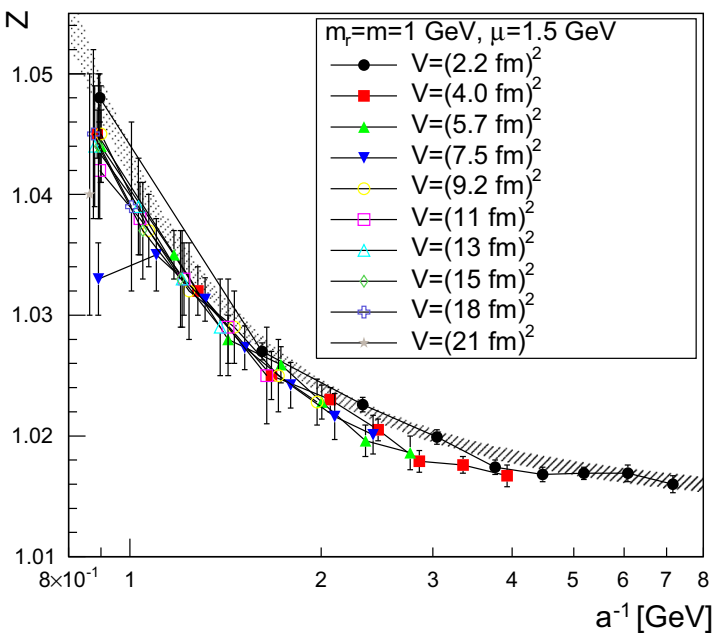

Fig. 3 The wave-function renormalization constant as a function of the lattice cutoff and the lattice volume in two dimensions for $\mu=1.5 \mathrm{GeV}$. The top-left panel shows the case of $m=m_{\mathrm{r}}=0 \mathrm{GeV}$, the top-right

All of this suggests to study the propagator of scalar matter in the quenched case to obtain further insights on the analytical structure, including the dependence on dimensionality. Thus, the aim of this work is to determine the quenched scalar fundamental propagator in a wide range of parameters and for two, three, and four dimensions, using lattice gauge theory. Having obtained the renormalized propagator, usage of the Schwinger function $[3,12]$ should then provide information on the analytic structure.

Besides these fundamental issues, the quenched calculation provides also an excellent testbed to study the lattice artifacts and renormalization properties of the scalar propagator beyond perturbation theory, which is helpful in studies of the dynamical case $[18,19,24]$.
Wave-function renormalization constant in two dimensions

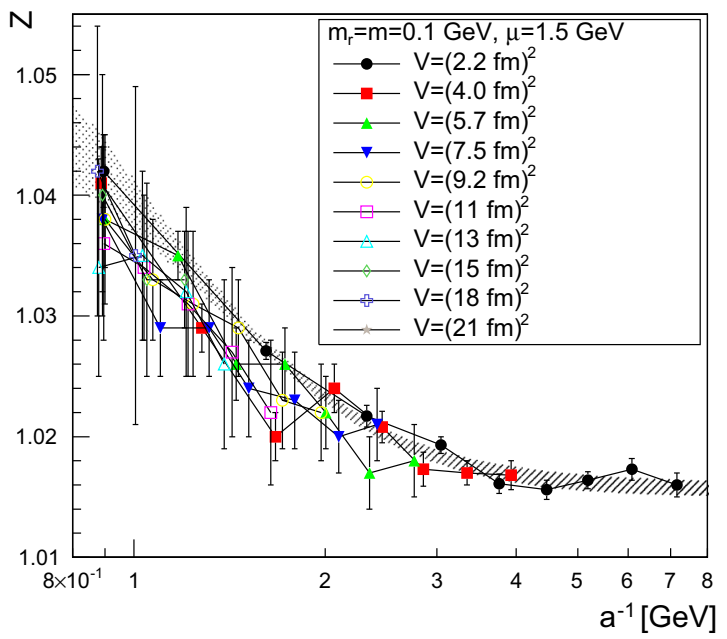

Wave-function renormalization constant in two dimensions

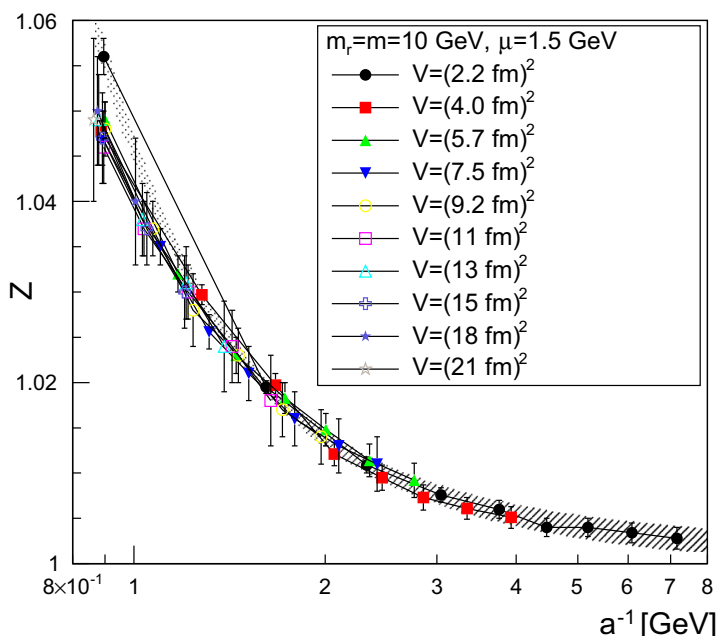

panel of $m=m_{\mathrm{r}}=0.1 \mathrm{GeV}$, the bottom-left panel of $m=m_{\mathrm{r}}=1 \mathrm{GeV}$, and the bottom-right panel of $m=m_{\mathrm{r}}=10 \mathrm{GeV}$. The hatched band is the fit (4) with the parameters given in Table 1

The technical setup of the calculations is presented in Sect. 2. The issue of renormalization is studied in detail in Sect. 3. The results in momentum space and for the analytical structure of the renormalized propagator are then presented in Sect. 4. These are the main results of this work. The findings are summarized in Sect. 5. Some preliminary results can be found in $[18,24]$.

\section{Technical setup}

In the following the propagator of a scalar particle in the fundamental representation in $\mathrm{SU}(2)$ in the quenched theory will be determined in two, three, and four dimensions. The 
Wave-function renormalization constant in three dimensions

N

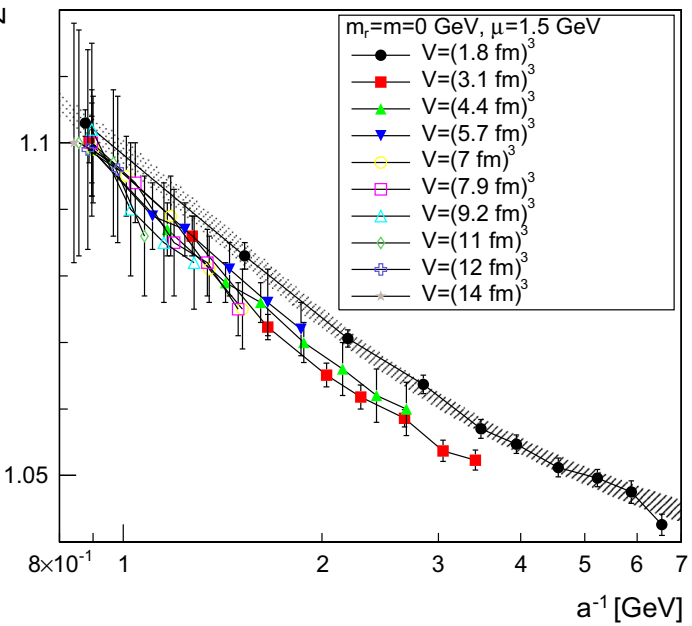

Wave-function renormalization constant in three dimensions

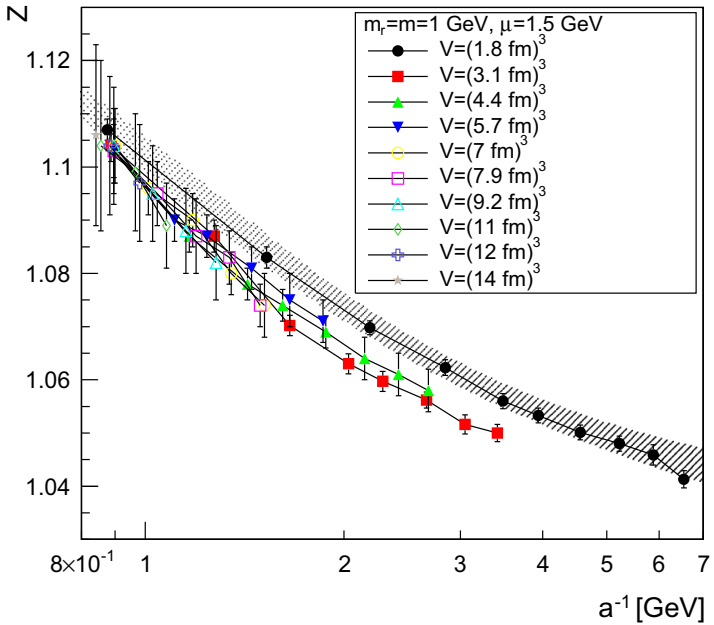

Fig. 4 The wave-function renormalization constant as a function of the lattice cutoff and the lattice volume in three dimensions for $\mu=$ $1.5 \mathrm{GeV}$. The top-left panel shows the case of $m=m_{\mathrm{r}}=0 \mathrm{GeV}$,

technical setup is based on [20,24,25]. Hence, the Wilson action for SU(2) Yang-Mills theory is simulated using a cycle of heatbath and overrelaxation updates. The lattice setups are listed in Table 3 in Appendix A. The determination of the lattice spacing has been performed as in [22].

Each configuration selected for measurement has been fixed to minimal Landau gauge [3] using adaptive stochastic overrelaxation [25]. The quenched fundamental propagator has been obtained as in [24]. In the continuum, it is given by the inverse of the covariant fundamental Laplacian including the mass term

$-D^{2}=-\left(\partial_{\mu}-i \frac{g \tau^{a}}{2} A_{\mu}^{a}\right)^{2}+m_{0}^{2}$,
Wave-function renormalization constant in three dimensions

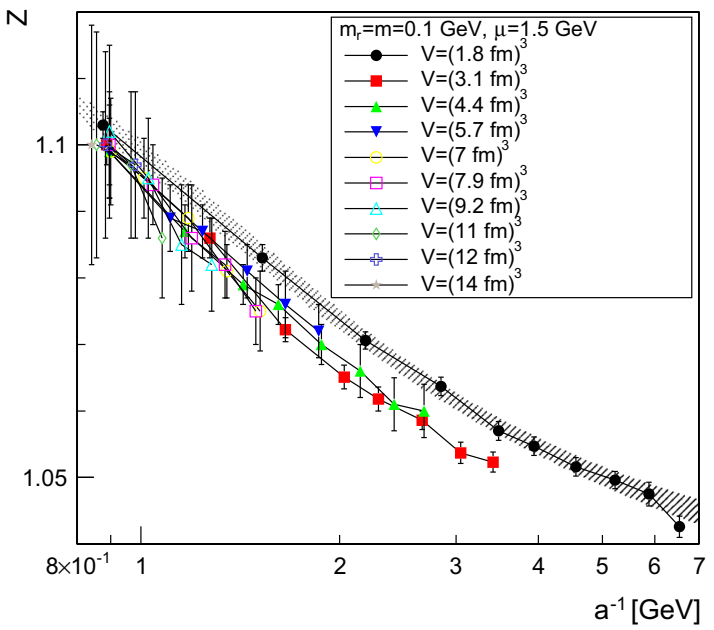

Wave-function renormalization constant in three dimensions

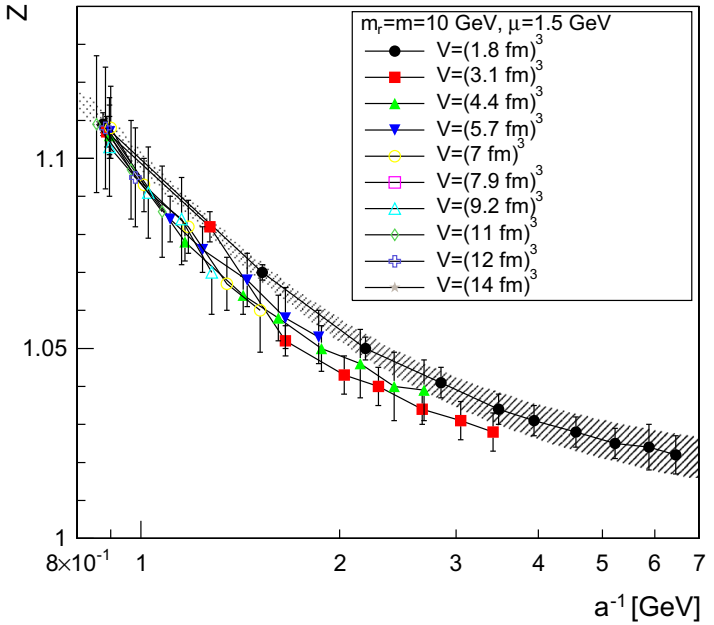

the top-right panel of $m=m_{\mathrm{r}}=0.1 \mathrm{GeV}$, the bottom-left panel of $m=m_{\mathrm{r}}=1 \mathrm{GeV}$, and the bottom-right panel of $m=m_{\mathrm{r}}=10 \mathrm{GeV}$. The hatched band is the fit (4) with the parameters given in Table 1

where the generators of the gauge algebra $\tau^{a}$ are the usual Pauli matrices, the $A_{\mu}^{a}$ are the gauge fields, $g=\sqrt{4 / \beta}$ the (bare) coupling constant, and $m_{0}$ the bare mass of the scalar. As the lattice version of this operator its naive discretization [26]

$$
\begin{aligned}
-D_{L}^{2}= & -\sum_{\mu}\left(U_{\mu}(x) \delta_{y\left(x+e_{\mu}\right)}+U_{\mu}^{\dagger}(x-\mu) \delta_{y\left(x-e_{\mu}\right)}-2 \delta_{x y}\right) \\
& +m_{0}^{2} \delta_{x y},
\end{aligned}
$$

has been used, where $U_{\mu}$ are the link variables and $e_{\mu}$ are lattice unit vectors in the corresponding directions. Since this operator is positive semi-definite, it can be inverted. This has been done using the same method as for the Faddeev-Popov operator in [25]. It should be noted that even a zero mass 

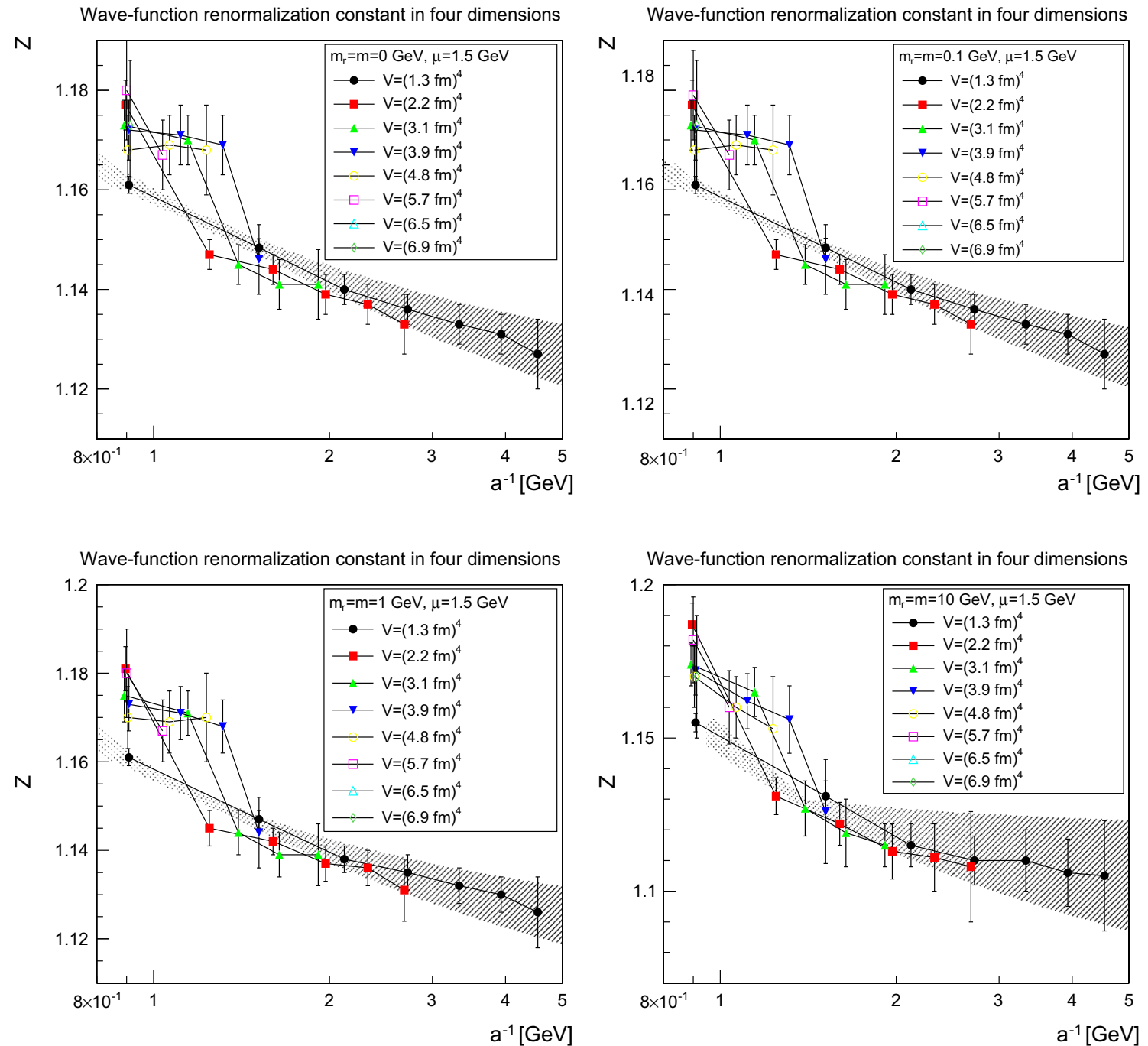

panel of $m=m_{\mathrm{r}}=0.1 \mathrm{GeV}$, the bottom-left panel of $m=m_{\mathrm{r}}=1 \mathrm{GeV}$, and the bottom-right panel of $m=m_{\mathrm{r}}=10 \mathrm{GeV}$. The hatched band is the fit (4) with the parameters given in Table 1
Fig. 5 The wave-function renormalization constant as a function of the lattice cutoff and the lattice volume in four dimensions for $\mu=1.5 \mathrm{GeV}$. The top-left panel shows the case of $m=m_{\mathrm{r}}=0 \mathrm{GeV}$, the top-right
Wave-function renormalization constant in four dimensions

Wave-function renormalization constant in four dimensions
Table 1 Fit parameters of (4) for the wave-function renormalization constants at $\mu=1.5 \mathrm{GeV}$. A value of 0 for $Z_{\infty}$ indicates that no stable fit with a non-zero value for $Z_{\infty}$ could be found

\begin{tabular}{llllll}
\hline$d$ & $m(\mathrm{GeV})$ & $Z_{\infty}$ & $\Lambda(\mathrm{GeV})$ & $\epsilon$ & $c$ \\
\hline 2 & 10 & $0.9991(16)$ & $1.103(5)$ & $1.58(7)$ & $0.0325(9)$ \\
2 & 1 & $1.0116(11)$ & $1.040(4)$ & $1.16(7)$ & $0.0214(2)$ \\
2 & 0.1 & $1.0157(8)$ & $2.2(4)$ & $5.2 \pm 1.5$ & $0.4_{-0.3}^{+1.9}$ \\
2 & 0 & $1.0157(8)$ & $2.1(5)$ & $5.1 \pm 1.7$ & $0.4_{-0.3}^{+2.1}$ \\
3 & 10 & $0.999(8)$ & $1.39(5)$ & $1.16(3)$ & $0.110(11)$ \\
3 & 1 & $1.00(19)$ & $1.4(3)$ & $0.7(6)$ & $0.105_{-0.004}^{+0.164}$ \\
3 & 0.1 & $1.00(6)$ & $1.48(15)$ & $0.6(4)$ & $0.11(4)$ \\
3 & 0 & $1.00(4)$ & $1.49(13)$ & $0.7(3)$ & $0.11(3)$ \\
4 & 10 & 0 & $0.8(5)$ & $1.129(7)$ & $0.021(17)$ \\
4 & 1 & 0 & $0.99(19)$ & $0.018(7)$ & $1.150(3)$ \\
4 & 0.1 & 0 & $1.10(18)$ & $0.020(7)$ & $1.153(3)$ \\
4 & 0 & 0 & $1.10(19)$ & $0.020(7)$ & $1.153(3)$ \\
\hline
\end{tabular}



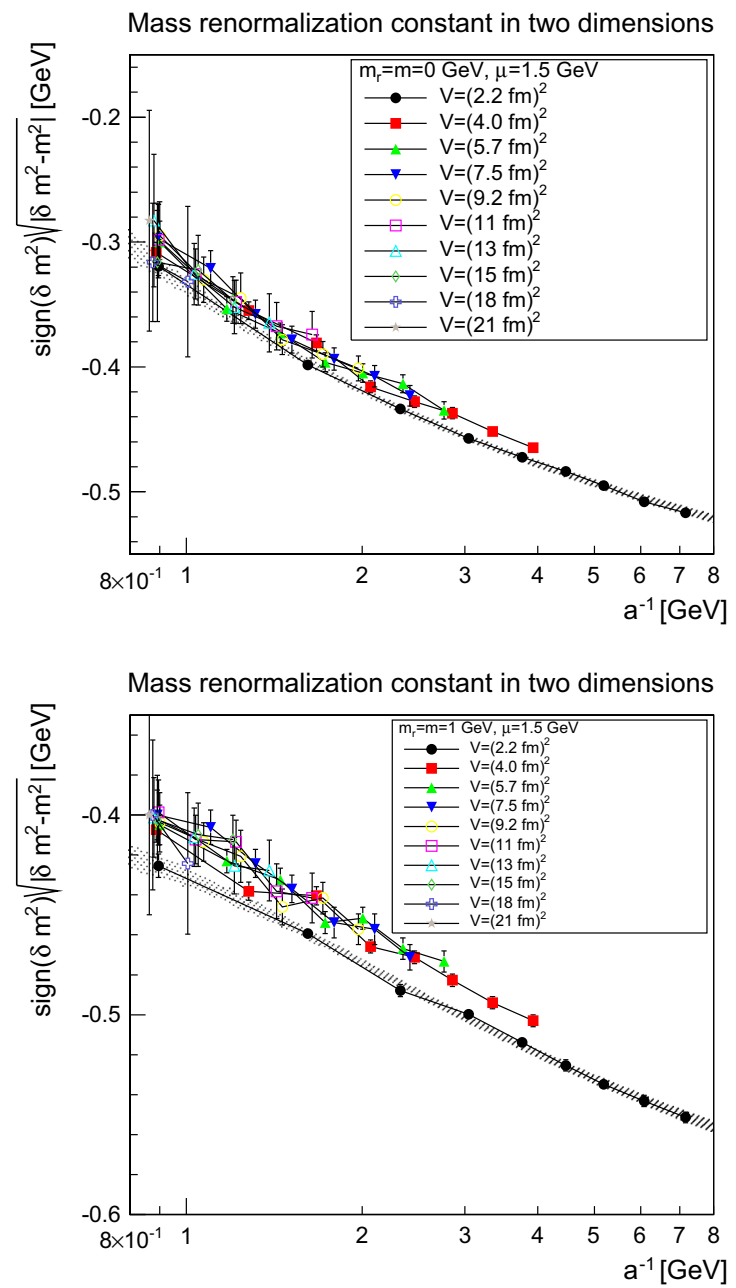

Fig. 6 The mass renormalization constant as a function of the lattice cutoff and the lattice volume in two dimensions for $\mu=1.5 \mathrm{GeV}$. The top-left panel shows the case of $m=m_{\mathrm{r}}=0 \mathrm{GeV}$, the top-right panel

is not a problem for this method. ${ }^{2}$ The final result has been averaged over color. The momenta have been evaluated along the $x$-axis as edge momenta and along the $x y, x y z$, and $x y z t$ diagonal directions, when available in a given number of dimensions.

This leaves to fix the bare mass $m_{0}$ in (1). Since the lattice spacings are known in advance, it can be set to the desired tree-level value $m=a m_{0}$ at the ultraviolet cutoff $1 / a$. Four different values will be used, zero, $100 \mathrm{MeV}, 1 \mathrm{GeV}$, and $10 \mathrm{GeV}$. The corresponding bare values $m_{0}$ for the case of $1 \mathrm{GeV}$ are listed in Table 3. While the values of $100 \mathrm{MeV}$ and $1 \mathrm{GeV}$ are suitable for the wide range of lattice parameters used here to expect no serious lattice artifacts, both zero mass

\footnotetext{
$\overline{2}$ In contrast to the Faddeev-Popov operator, this operator has no trivial zero modes, and thus an inversion even at zero momentum is possible. However, since constant modes affect the result on a finite lattice, this is not done here.
}
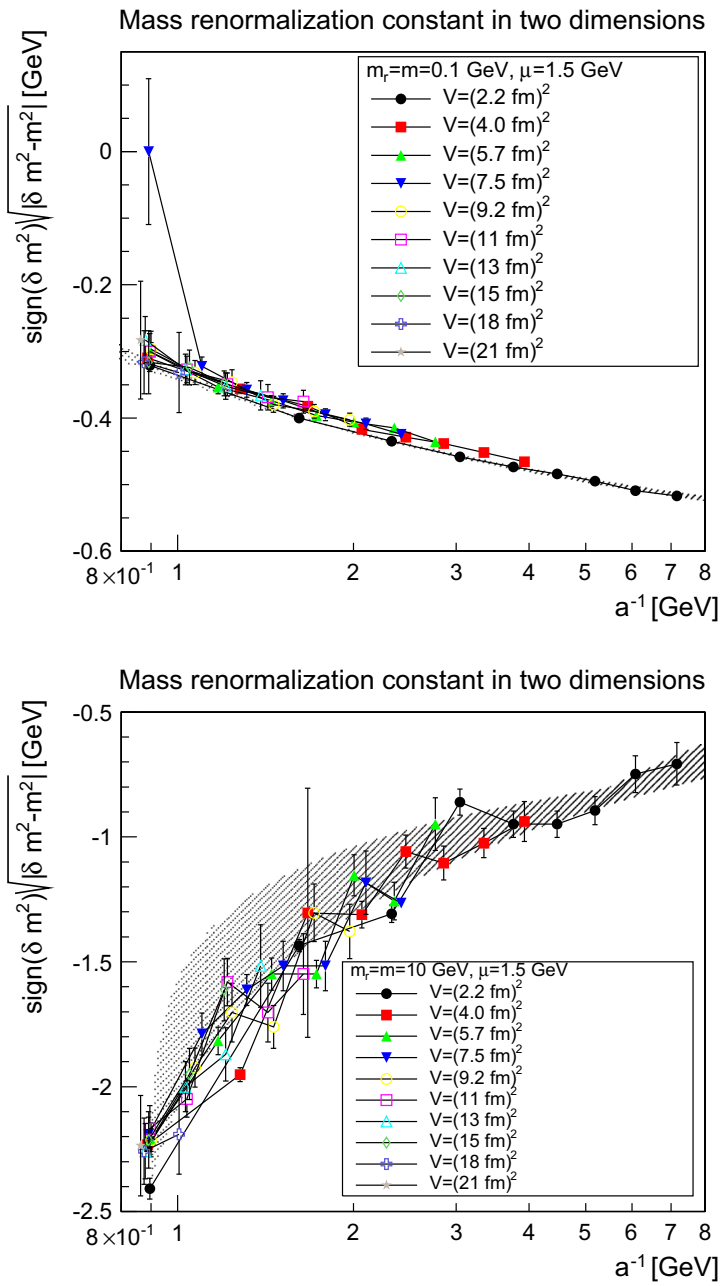

of $m=m_{\mathrm{r}}=0.1 \mathrm{GeV}$, the bottom-left panel of $m=m_{\mathrm{r}}=1 \mathrm{GeV}$, and the bottom-right panel of $m=m_{\mathrm{r}}=10 \mathrm{GeV}$. The hatched band is the fit (5) with the parameters given in Table 2

and $10 \mathrm{GeV}$ are naively expected to be strongly affected by lattice artifacts, and therefore are intended to serve as benchmarks for these artifacts. However, it will turn out that these artifacts are often surprisingly small.

\section{Renormalization}

\subsection{Definition of the scheme}

Assuming that the renormalization of the propagator can be performed as in the perturbative case [27], which will be supported by the results, there are two necessary renormalization constants. One is a multiplicative wave-function renormalization $Z$, and one an additive mass renormalization $\delta m^{2}$, leading to the renormalized propagator

$D^{i j}\left(p^{2}\right)=\frac{\delta^{i j}}{Z\left(p^{2}+m_{\mathrm{r}}^{2}\right)+\Pi\left(p^{2}\right)+\delta m^{2}}$, 

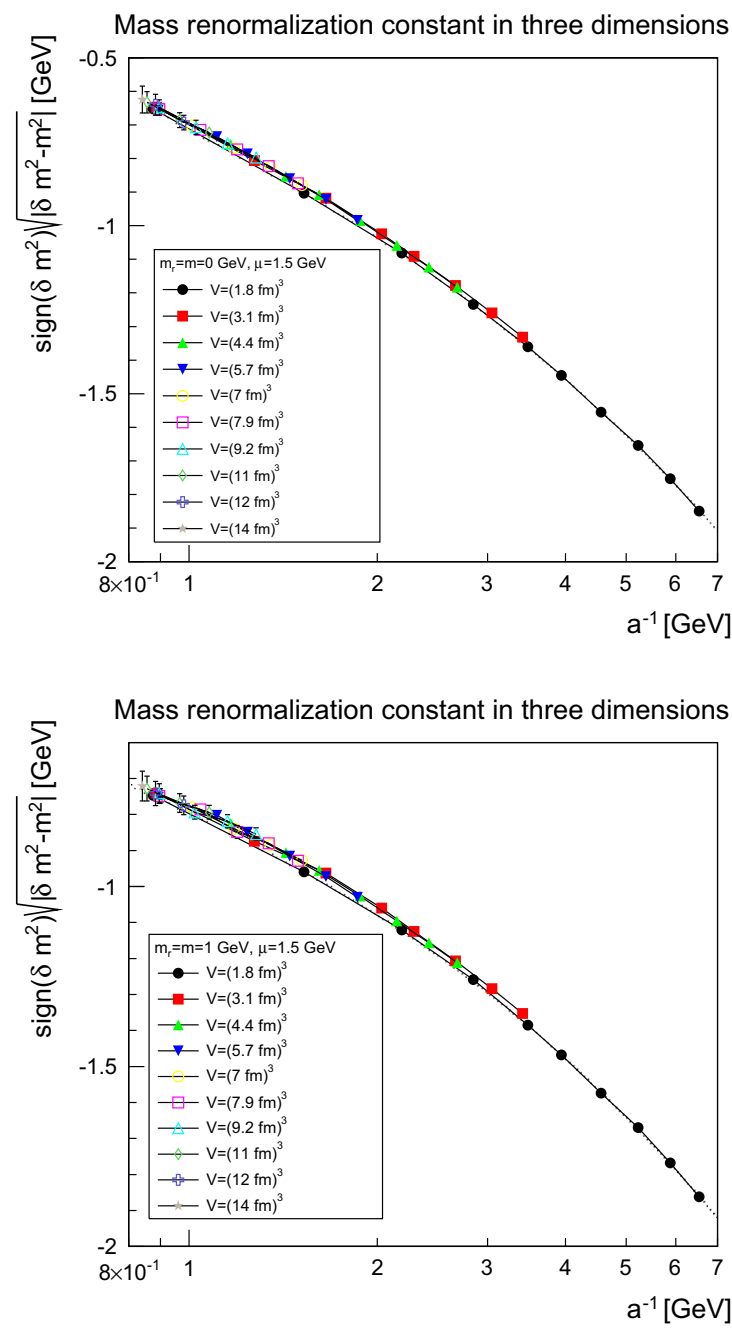

Fig. 7 The mass renormalization constant as a function of the lattice cutoff and the lattice volume in three dimensions for $\mu=1.5 \mathrm{GeV}$. The top-left panel shows the case of $m=m_{\mathrm{r}}=0 \mathrm{GeV}$, the top-right panel of $m=m_{\mathrm{r}}=0.1 \mathrm{GeV}$, the bottom-left panel of $m=m_{\mathrm{r}}=1 \mathrm{GeV}$, and

where $m_{\mathrm{r}}^{2}$ is the renormalized mass, $p^{2}$ is the momentum, and $\Pi\left(p^{2}\right)$ is the self-energy obtained from the unrenormalized color-averaged propagator $D_{\mathrm{u}}=D_{\mathrm{u}}^{i i} / N_{\mathrm{c}}$,

$\Pi\left(p^{2}\right)=\frac{1-p^{2} D_{\mathrm{u}}\left(p^{2}\right)}{D_{\mathrm{u}}\left(p^{2}\right)}$,

and therefore it encodes the deviation from the tree-level propagator as

$D_{\mathrm{u}}=\frac{1}{p^{2}+\Pi\left(p^{2}\right)}$.

The inclusion of the tree-level mass $m^{2}$ in the self-energy is for technical convenience only, as it avoids to involve explicitly the scale $a$. This only shifts the renormalization constant $\delta m^{2}$ by the tree-level mass $m^{2}$, and it has no other implications.
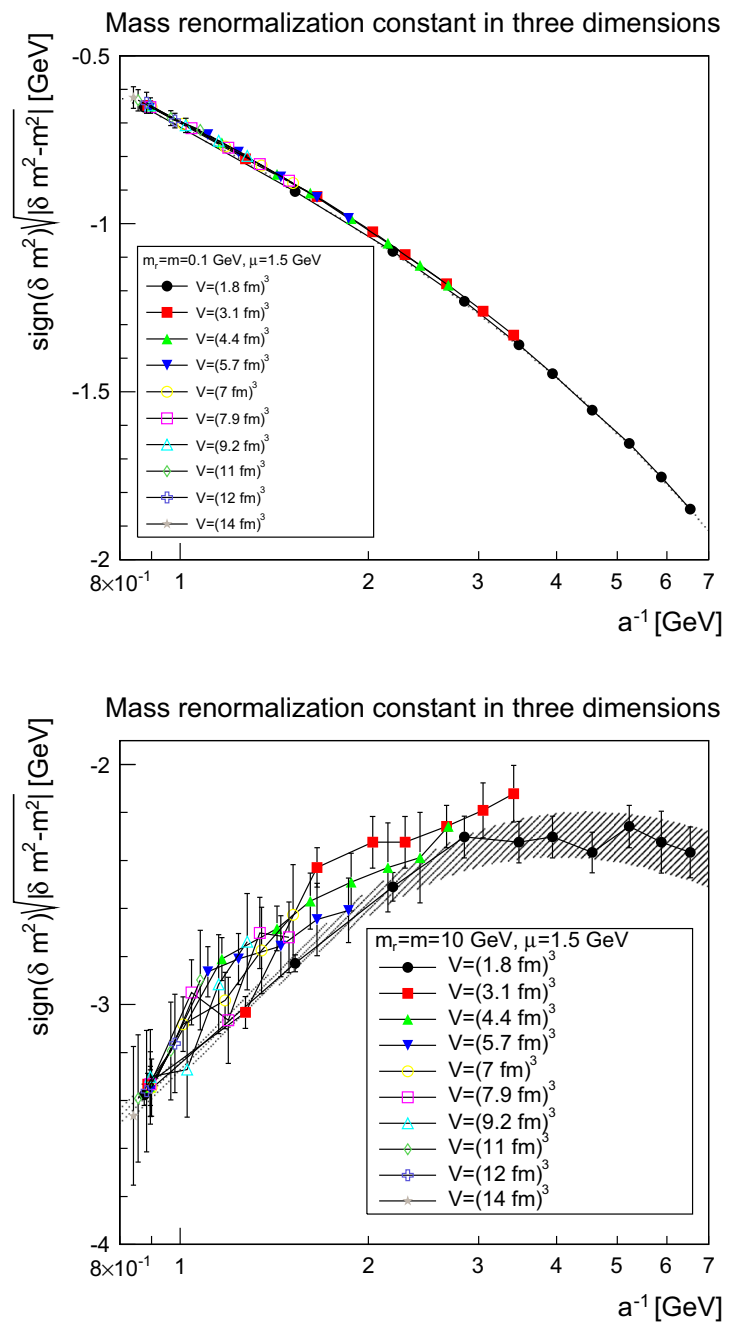

the bottom-right panel of $m=m_{\mathrm{r}}=10 \mathrm{GeV}$. The hatched band is the fit (5) with the parameters given in Table 2. Note that the hatched band can be as narrow as the lines, and therefore not be visible

The renormalization scheme to fix both renormalization constants is

$$
\begin{aligned}
D^{i j}\left(\mu^{2}\right) & =\frac{\delta^{i j}}{\mu^{2}+m_{\mathrm{r}}^{2}} \\
\frac{\partial D^{i j}}{\partial p}\left(\mu^{2}\right) & =-\frac{2 \mu \delta^{i j}}{\left(\mu^{2}+m_{\mathrm{r}}^{2}\right)^{2}},
\end{aligned}
$$

with the renormalization scale $\mu$. This requires the propagator and its derivative to have the tree-level values at $p^{2}=\mu^{2}$. In most of the paper the choice $\mu=1.5 \mathrm{GeV}$ and $m_{\mathrm{r}}=m$ will be made. The effect of different choices will be investigated in Sects. 3.3 and 4.3.

Solving for the renormalization constants, this yields

$$
Z=\frac{2 \mu-\frac{\mathrm{d} \Pi\left(p^{2}\right)}{\mathrm{d} p}\left(\mu^{2}\right)}{2 \mu}
$$



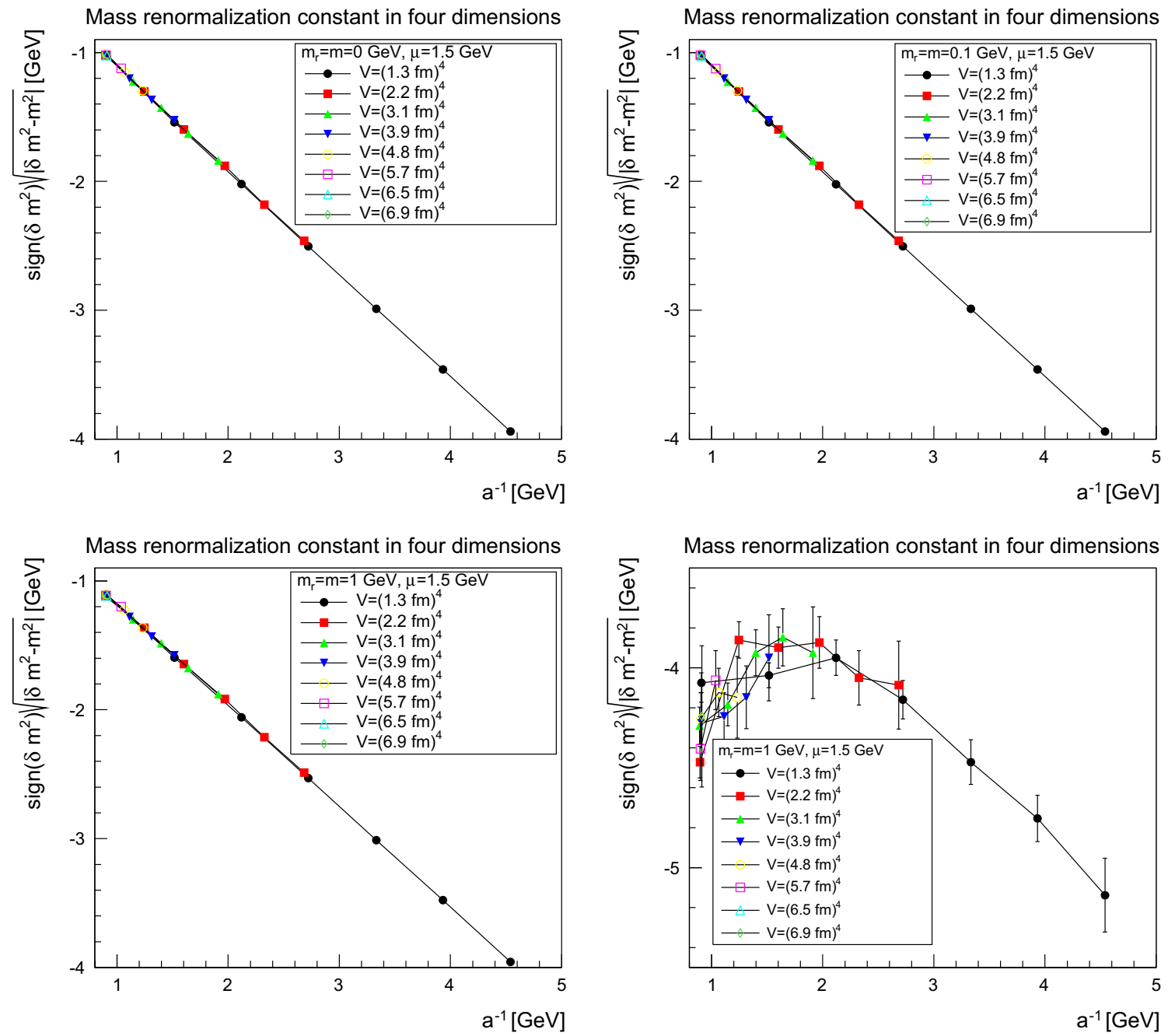

Fig. 8 The mass renormalization constant as a function of the lattice cutoff and the lattice volume in four dimensions for $\mu=1.5 \mathrm{GeV}$. The top-left panel shows the case of $m=m_{\mathrm{r}}=0 \mathrm{GeV}$, the top-right panel of $m=m_{\mathrm{r}}=0.1 \mathrm{GeV}$, the bottom-left panel of $m=m_{\mathrm{r}}=1 \mathrm{GeV}$,

and the bottom-right panel of $m=m_{\mathrm{r}}=10 \mathrm{GeV}$. The hatched band is the fit (5) with the parameters given in Table 2. Note that the hatched band can be as narrow as the lines and therefore is not visible. No fit was possible using this fit form for $m=m_{\mathrm{r}}=10 \mathrm{GeV}$

Table 2 Fit parameters of (5) for the mass renormalization constants at $\mu=1.5 \mathrm{GeV}$. Note that a fit for $m=10 \mathrm{GeV}$ in four dimensions was not possible using this fit form

\begin{tabular}{llllll}
\hline$d$ & $m(\mathrm{GeV})$ & $\Lambda(\mathrm{GeV})$ & $\epsilon$ & $\delta$ & $c(\mathrm{GeV})$ \\
\hline 2 & 10 & $0.62(18)$ & $0.06(4)$ & 0 & $8.5(4)$ \\
2 & 1 & $1.47(9)$ & $0.065(3)$ & 0 & $-1.4179(16)$ \\
2 & 0.1 & $0.91(6)$ & $0.182(3)$ & 0 & $-0.4792(11)$ \\
2 & 0 & $0.89(6)$ & $0.223(3)$ & 0 & $-0.3793(11)$ \\
3 & 10 & $1.150(19)$ & $0.383(11)$ & $-0.2409(7)$ & $7.209(4)$ \\
3 & 1 & $1.142(4)$ & $0.1916(8)$ & $0.4018(3)$ & $-1.734(2)$ \\
3 & 0.1 & $1.950(15)$ & $-0.267(16)$ & $0.588(9)$ & $-0.906(5)$ \\
3 & 0 & $0.610(8)$ & $0.034(3)$ & $0.4603(16)$ & $-0.7428(15)$ \\
4 & 1 & $1.086(3)$ & $-0.596(11)$ & $1.082(8)$ & $-1.885(3)$ \\
4 & 0.1 & $1.110(5)$ & $-0.319(11)$ & $1.083(8)$ & $-1.1257(18)$ \\
4 & 0 & $1.139(6)$ & $-0.2722(12)$ & $1.076(9)$ & $-1.0520(18)$ \\
\hline
\end{tabular}



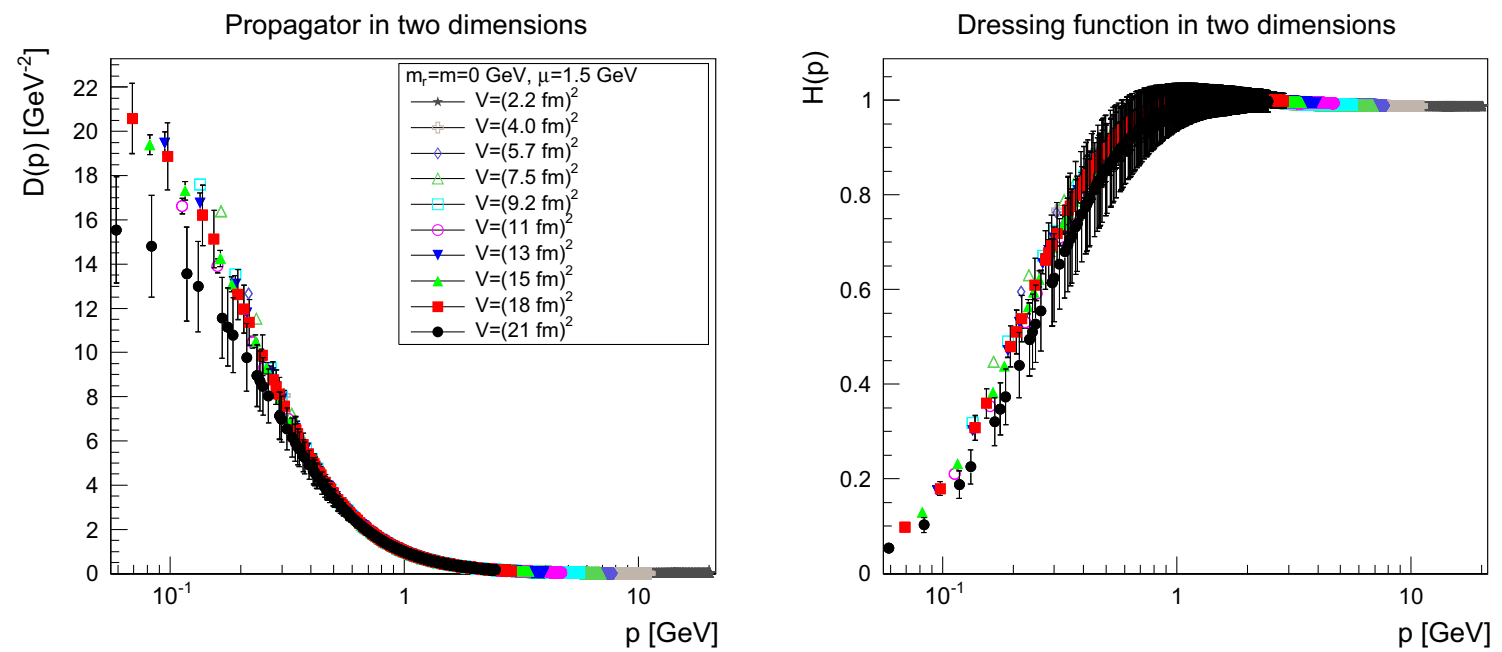

Fig. 9 The propagator (left panel) and the dressing function (6) (right panel) in two dimensions for $m=m_{\mathrm{r}}=0 \mathrm{GeV}$ and $\mu=1.5 \mathrm{GeV}$
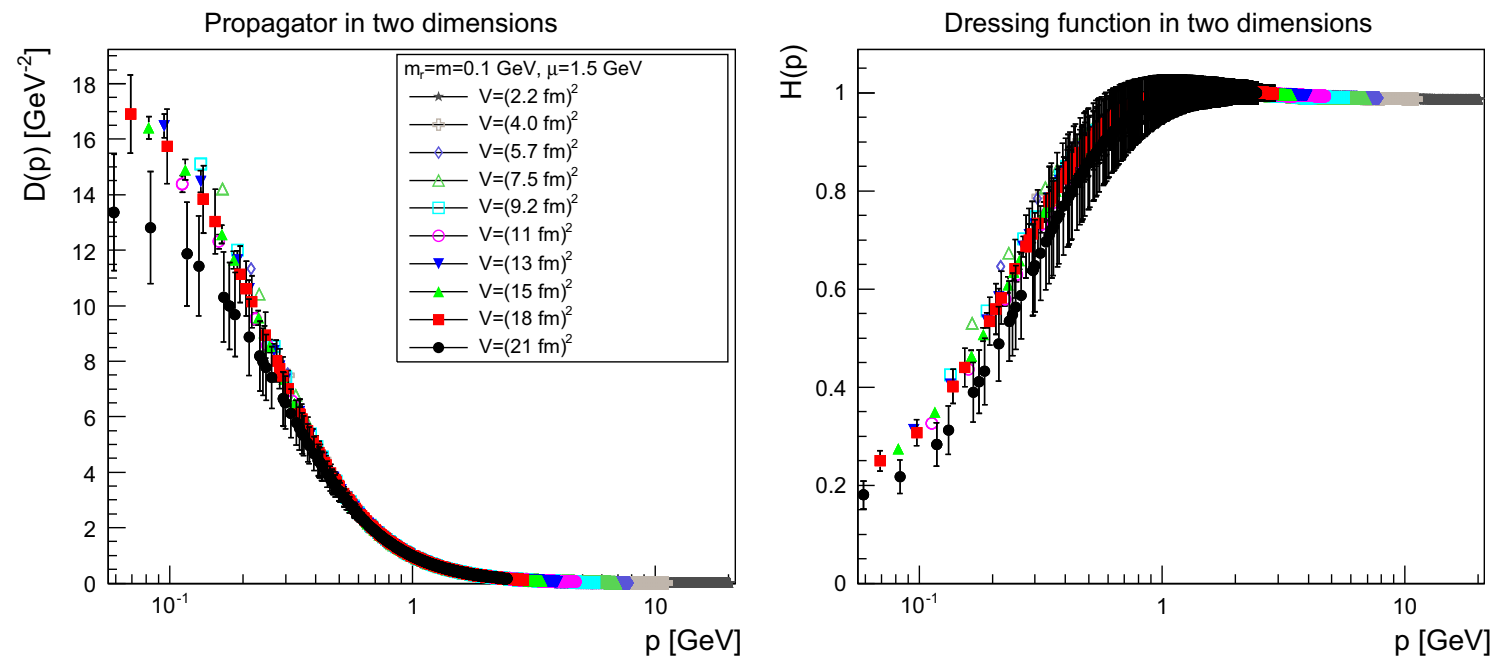

Fig. 10 The propagator (left panel) and the dressing function (6) (right panel) in two dimensions for $m=m_{\mathrm{r}}=0.1 \mathrm{GeV}$ and $\mu=1.5 \mathrm{GeV}$
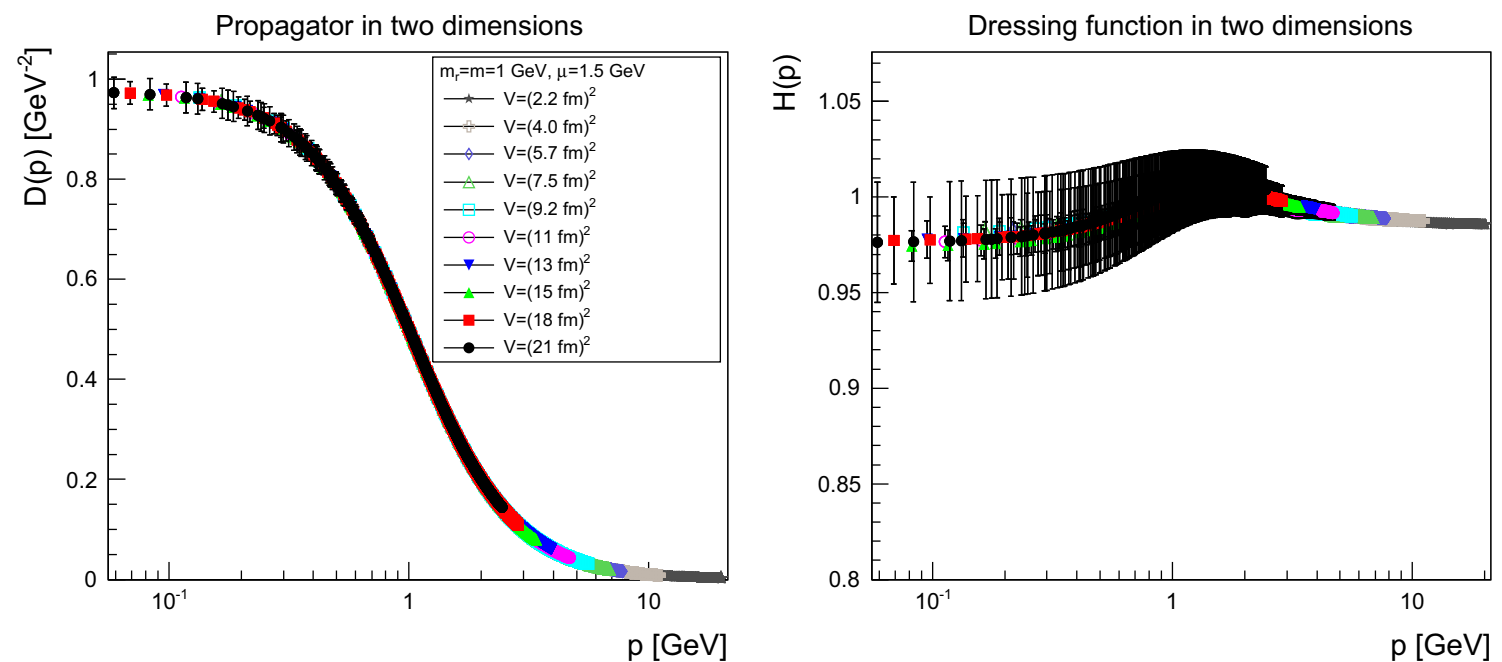

Fig. 11 The propagator (left panel) and the dressing function (6) (right panel) in two dimensions for $m=m_{\mathrm{r}}=1 \mathrm{GeV}$ and $\mu=1.5 \mathrm{GeV}$. Note the different scale in the right-hand panel compared to Figs. 9 and 10 

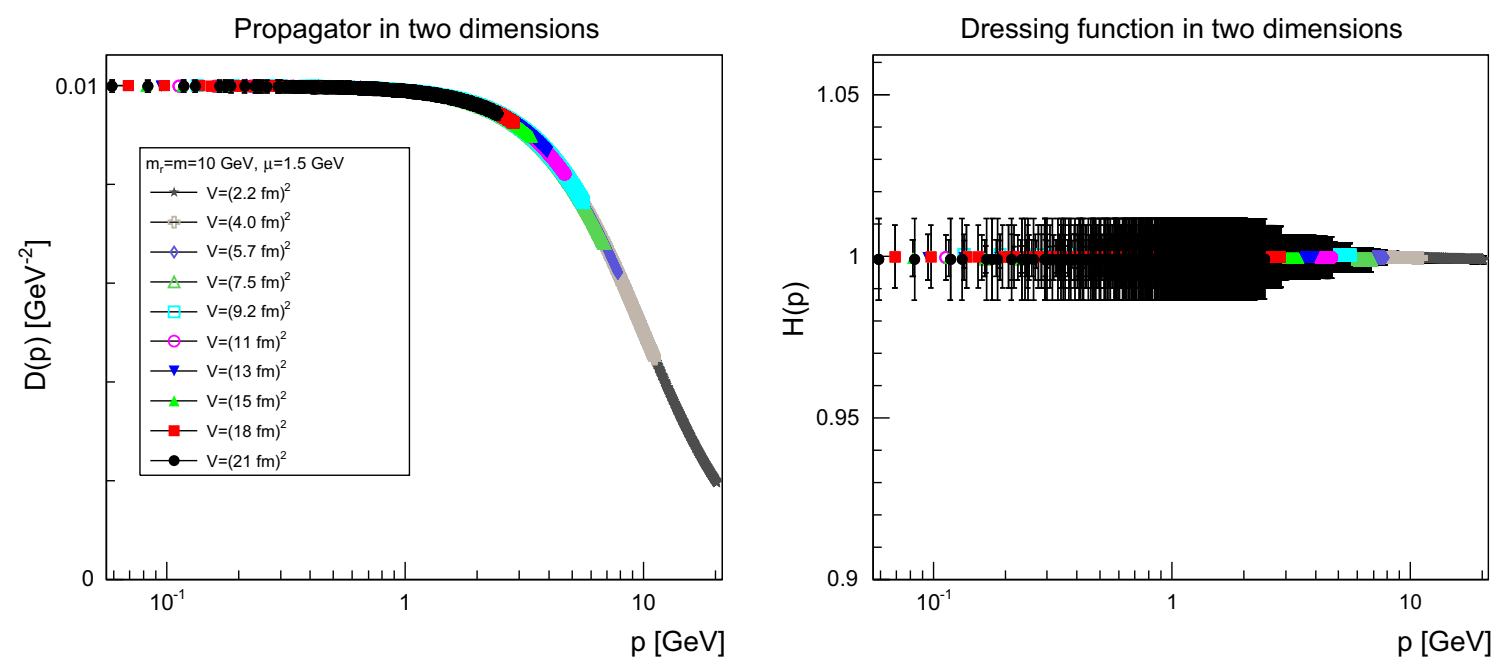

Fig. 12 The propagator (left panel) and the dressing function (6) (right panel) in two dimensions for $m=m_{\mathrm{r}}=10 \mathrm{GeV}$ and $\mu=1.5 \mathrm{GeV}$. Note the different scale in the right-hand panel compared to Figs. 9 and 10
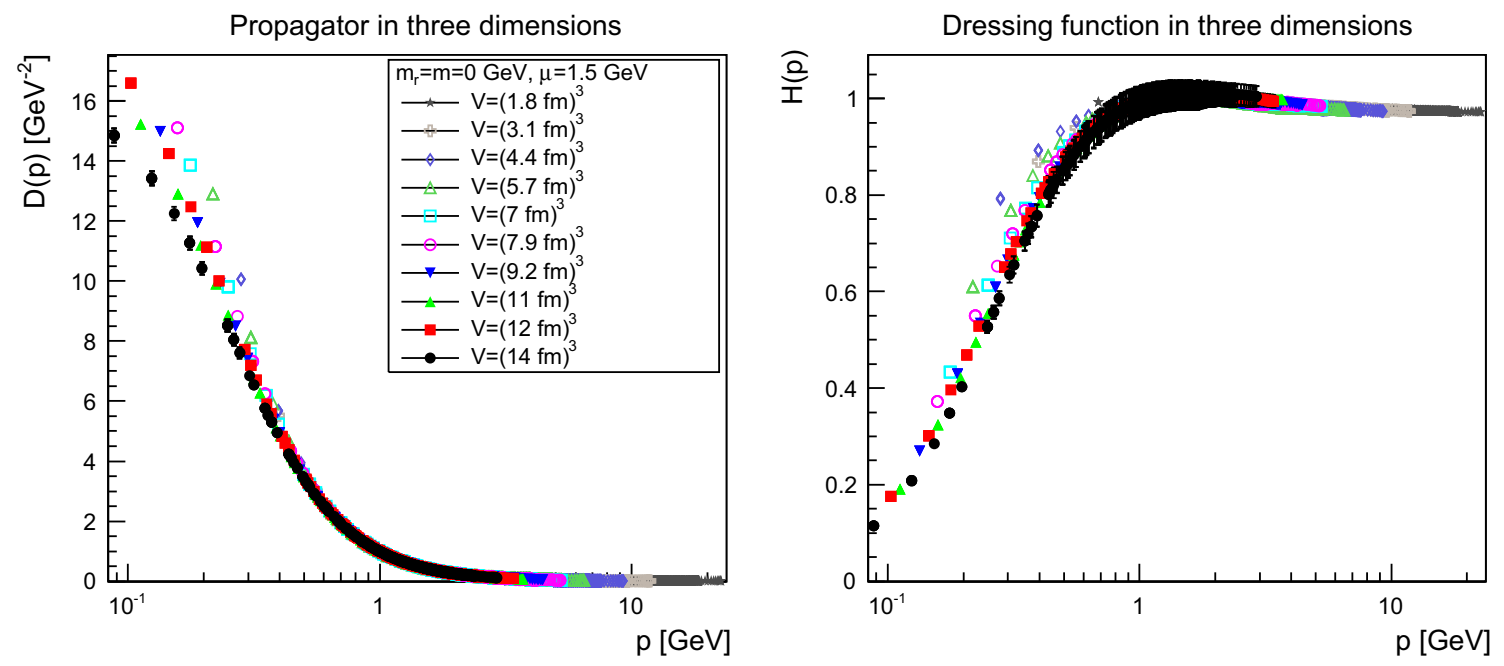

Fig. 13 The propagator (left panel) and the dressing function (6) (right panel) in three dimensions for $m=m_{\mathrm{r}}=0 \mathrm{GeV}$ and $\mu=1.5 \mathrm{GeV}$
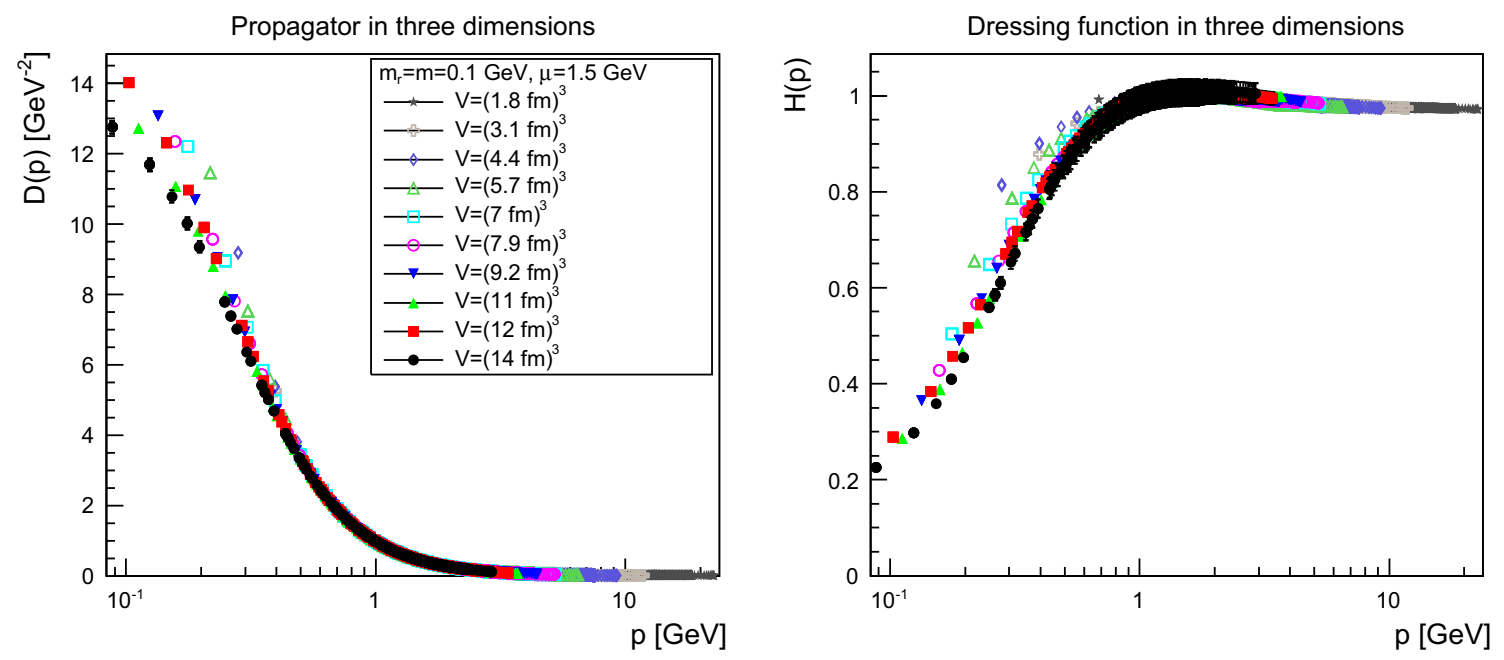

Fig. 14 The propagator (left panel) and the dressing function (6) (right panel) in three dimensions for $m=m_{\mathrm{r}}=0.1 \mathrm{GeV}$ and $\mu=1.5 \mathrm{GeV}$ 

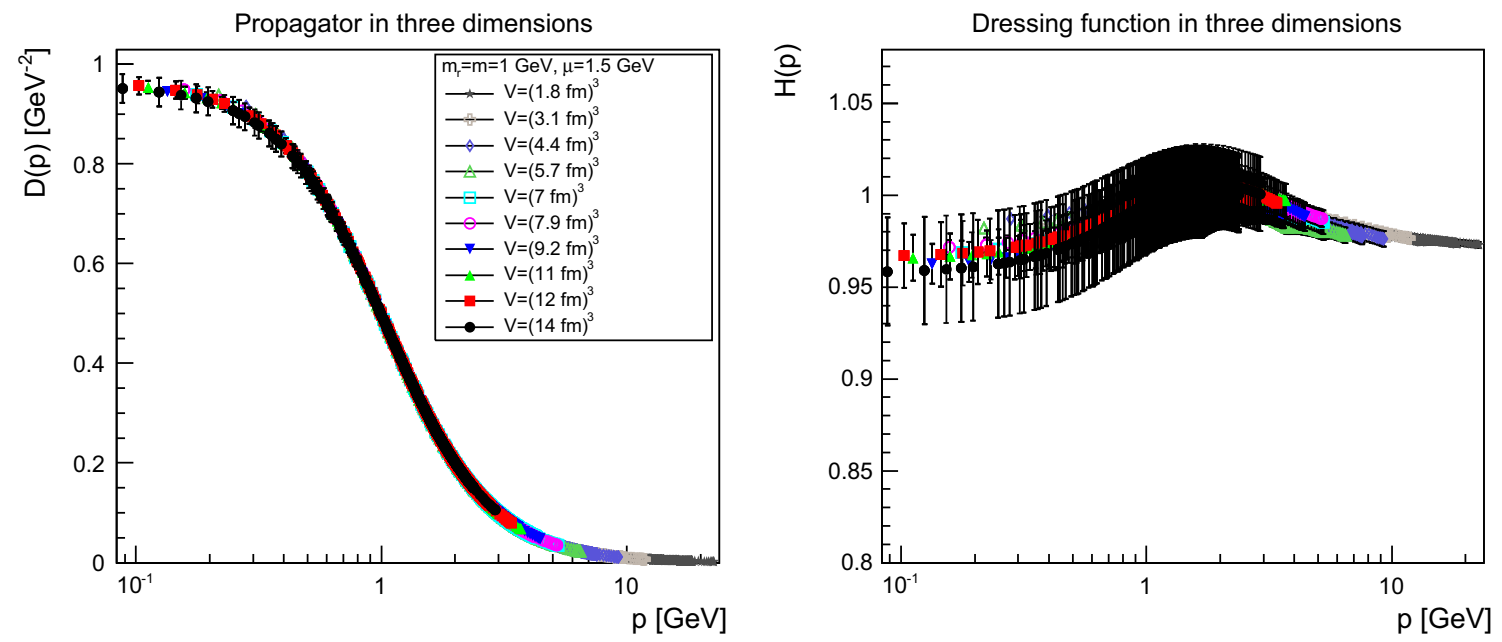

Fig. 15 The propagator (left panel) and the dressing function (6) (right panel) in three dimensions for $m=m_{\mathrm{r}}=1 \mathrm{GeV}$ and $\mu=1.5 \mathrm{GeV}$. Note the different scale in the right-hand panel compared to Figs. 13 and 14
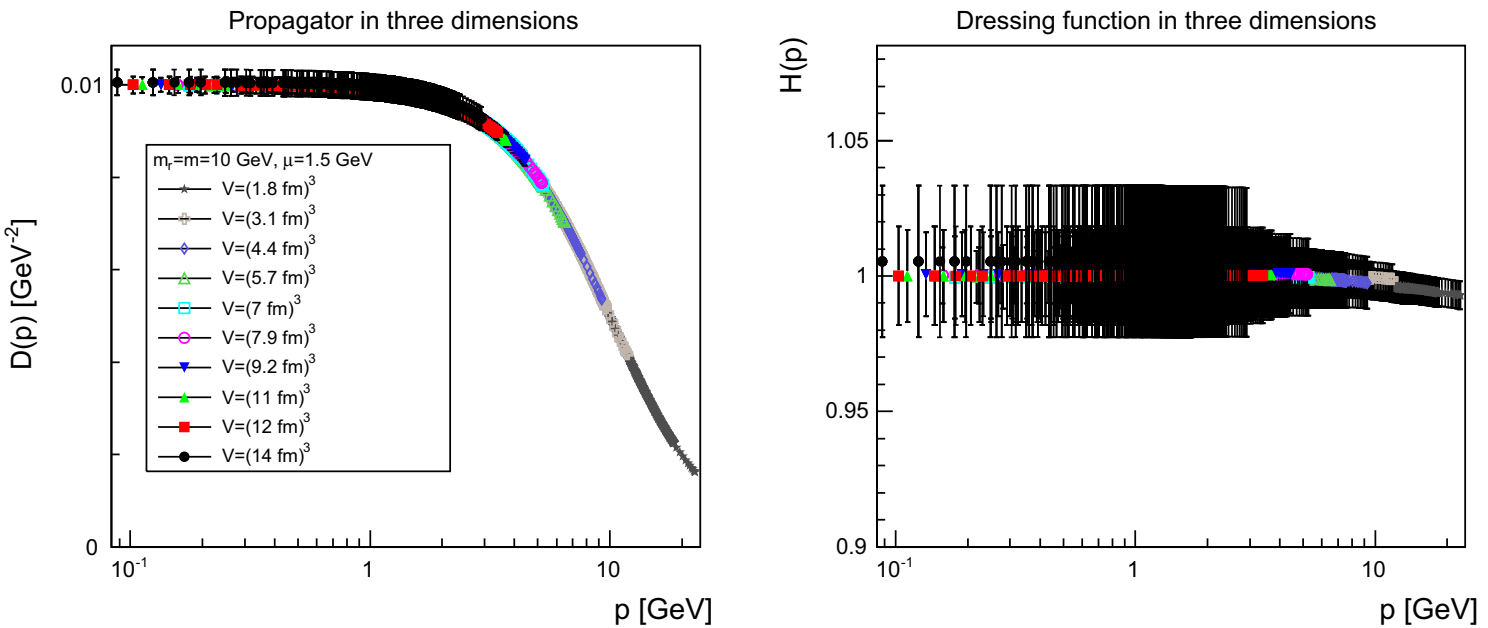

Fig. 16 The propagator (left panel) and the dressing function (6) (right panel) in three dimensions for $m=m_{\mathrm{r}}=10 \mathrm{GeV}$ and $\mu=1.5 \mathrm{GeV}$. Note the different scale in the right-hand panel compared to Figs. 13 and 14
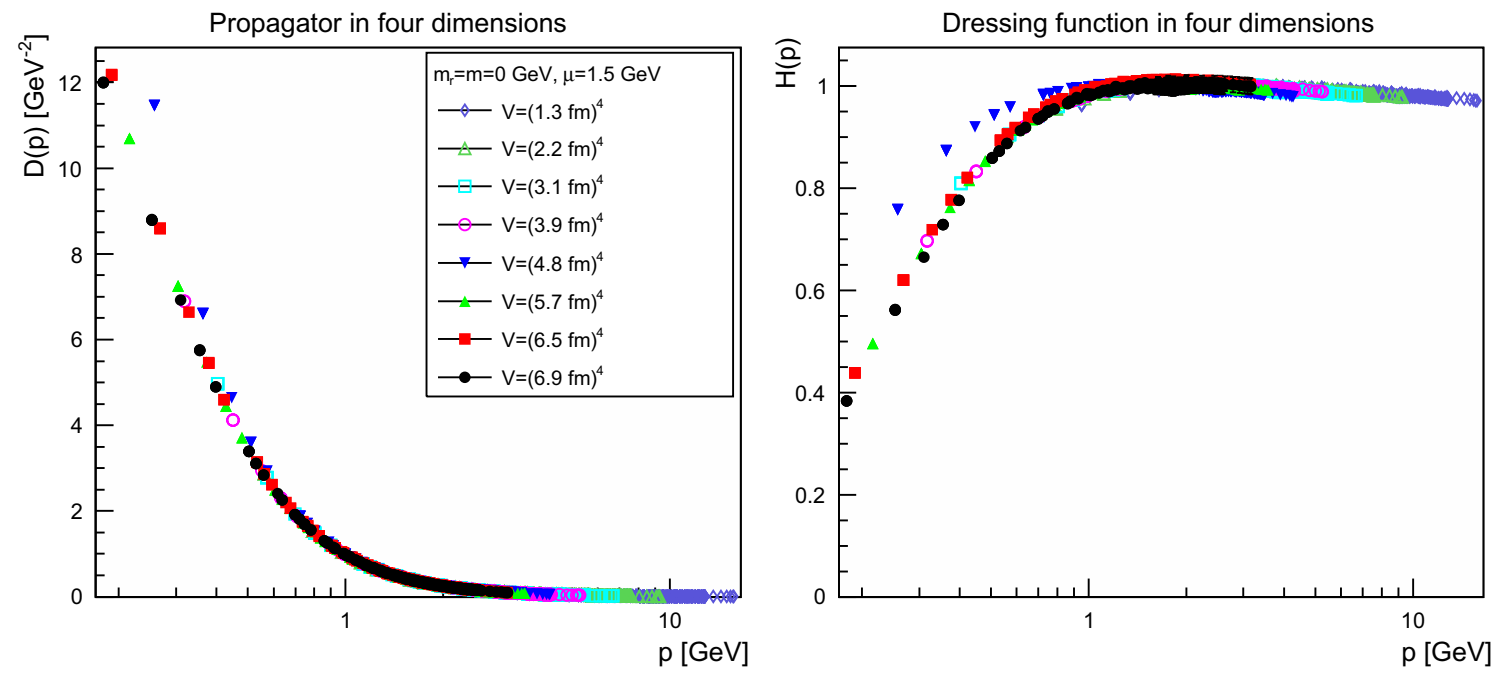

Fig. 17 The propagator (left panel) and the dressing function (6) (right panel) in four dimensions for $m=m_{\mathrm{r}}=0 \mathrm{GeV}$ and $\mu=1.5 \mathrm{GeV}$ 

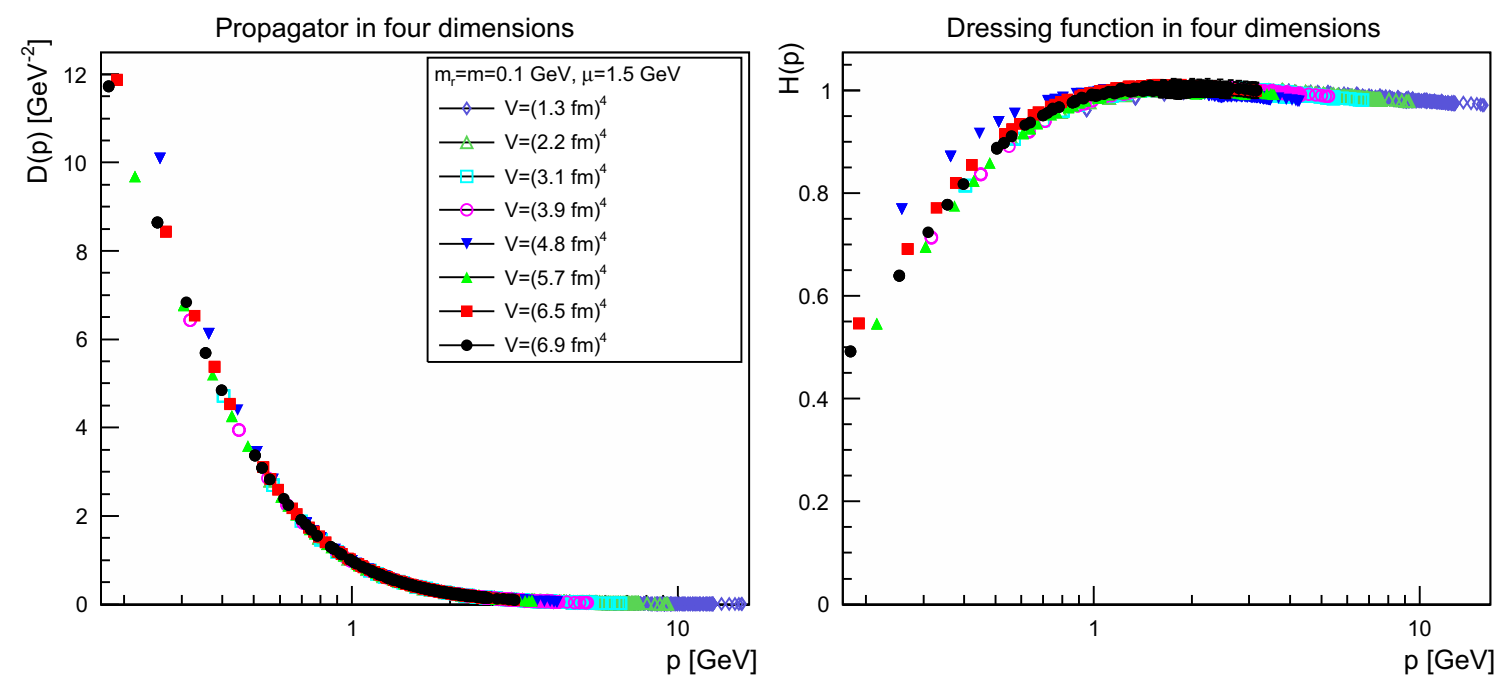

Fig. 18 The propagator (left panel) and the dressing function (6) (right panel) in four dimensions for $m=m_{\mathrm{r}}=0.1 \mathrm{GeV}$ and $\mu=1.5 \mathrm{GeV}$
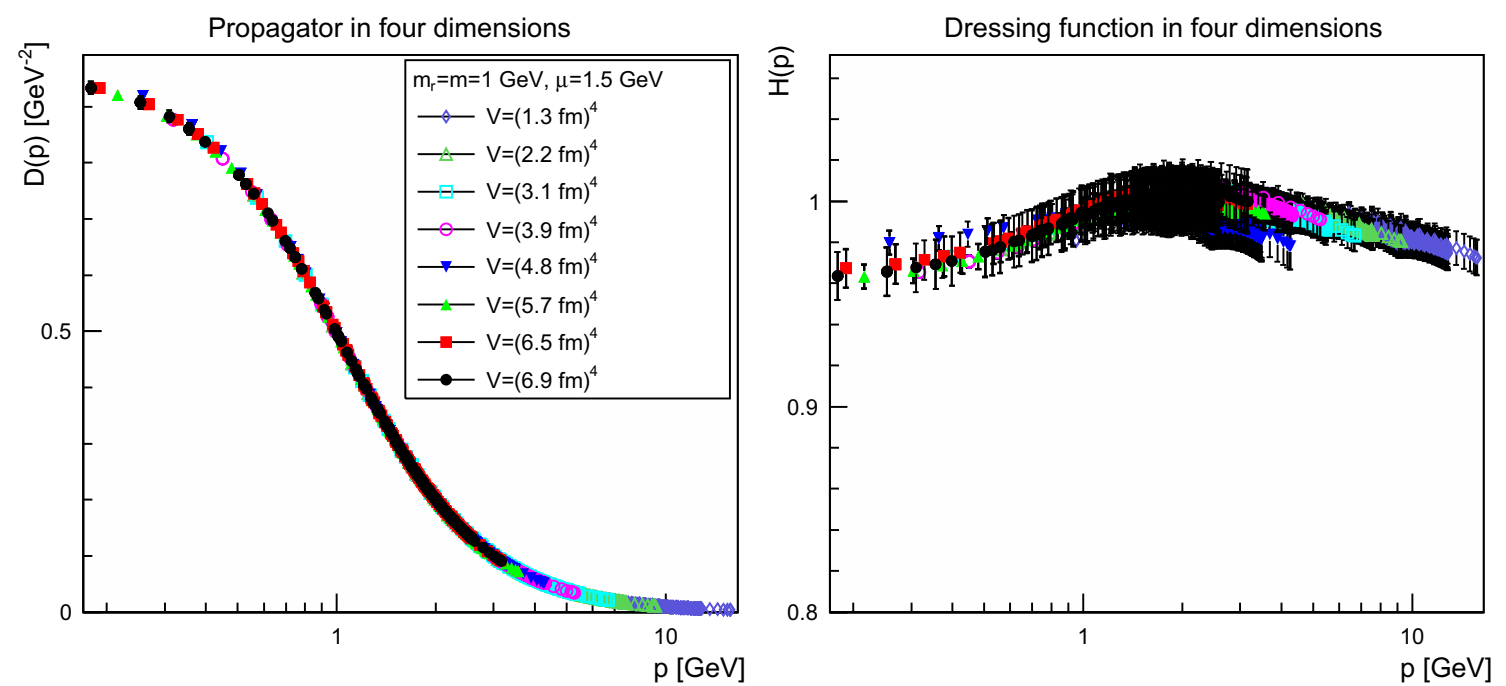

Fig. 19 The propagator (left panel) and the dressing function (6) (right panel) in four dimensions for $m=m_{\mathrm{r}}=1 \mathrm{GeV}$ and $\mu=1.5 \mathrm{GeV}$. Note the different scale in the right-hand panel compared to Figs. 17 and 18

$\delta m^{2}=\frac{\left(\mu^{2}+m_{\mathrm{r}}^{2}\right) \frac{\mathrm{d} \Pi\left(p^{2}\right)}{\mathrm{d} p}\left(\mu^{2}\right)-2 \mu \Pi\left(\mu^{2}\right)}{2 \mu}$.

Numerically, these constants are determined by linear interpolation between the two momenta values along the $x$-axis between which the actual value of $\mu$ is. The derivative of $\Pi$ is obtained by deriving the linear interpolation of $\Pi$ between both points analytically. Errors are then determined by error propagation from the original propagator, whose statistical error in turn has been determined using bootstrap [25]. Note that throughout only values for $\mu$ have been chosen such that the lower of the two momentum values has been non-zero, and the higher of the two momentum values has not been the maximum momentum along the $x$-axis of $2 / a$.
Using the derivative with respect to $p$ rather than $p^{2}$ for the discretization is numerically convenient, as the lattice momentum in the relevant region are approximately linearly spaced, at least for moderately small discretizations. Of course, this is completely equivalent to the more conventional derivative with respect to $p^{2}$.

\subsection{Numerical results and discretization dependence}

The effect of renormalization is shown in Fig. 1. It is visible that for all dimensions, though only very slightly in two dimensions, the propagators deviate from each other for different values of $a$. This deviation is not only a multiplica- 

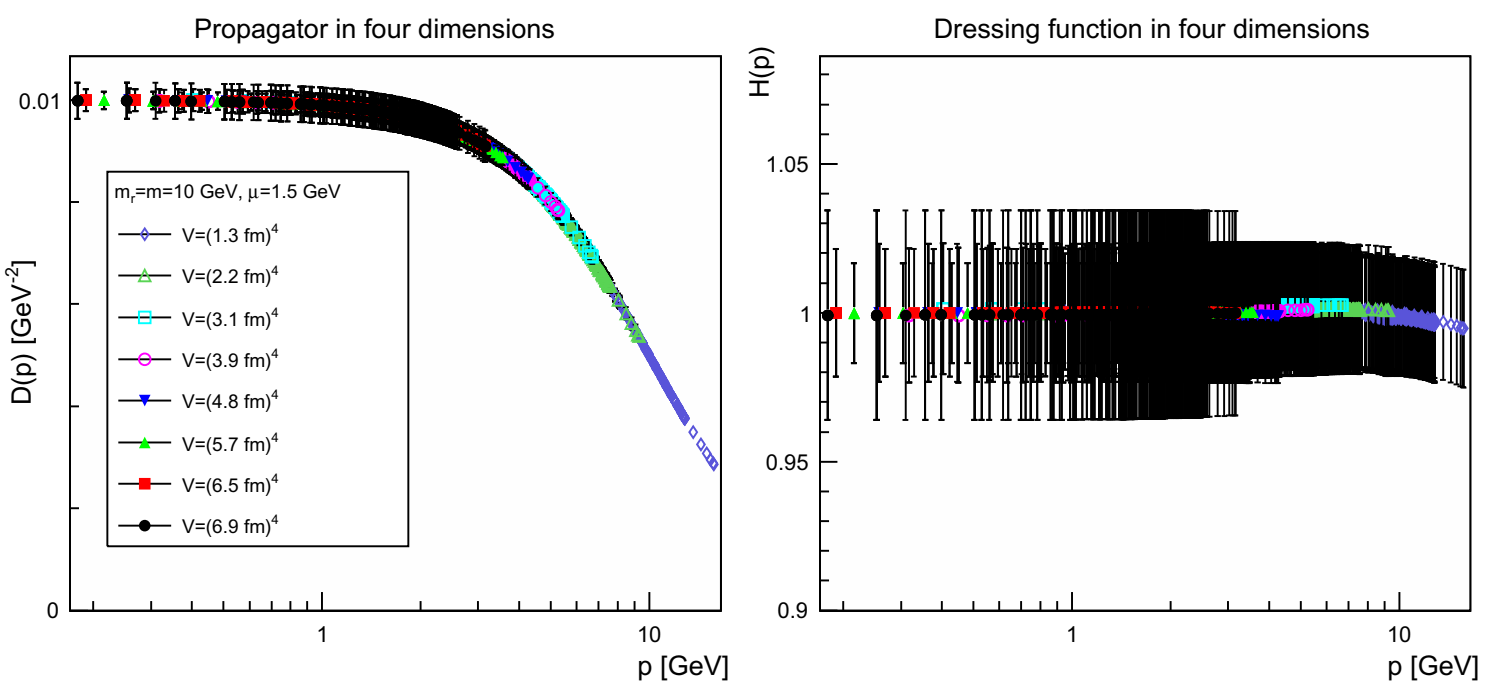

Fig. 20 The propagator (left panel) and the dressing function (6) (right panel) in four dimensions for $m=m_{\mathrm{r}}=10 \mathrm{GeV}$ and $\mu=1.5 \mathrm{GeV}$. Note the different scale in the right-hand panel compared to Figs. 17 and 18

tive factor, as they still coincide in the ultraviolet, but also a change of mass as the difference in the infrared shows. In fact, the results already suggest, and this will be confirmed below, that the wave-function renormalization is close to one, while the mass renormalization is sizable. After renormalization, the propagators show essentially no difference anymore, and thus only a very weak dependence on the lattice spacing. The same pattern is observed for the other masses in principle, but altered by two effects. For the smaller masses, as will be seen, finite-volume effects play a role in the infrared. For the larger mass, the propagator is very close to tree-level for almost all momenta accessible and the effects of renormalization are therefore substantially suppressed. Nonetheless, in all cases the renormalized propagators essentially coincide.

This also shows that there are only rather small discretization artifacts present after renormalization. This will be discussed more in detail in Sect. 3.4, where the volume and cutoff dependence of the renormalization constants will be analyzed.

However, there is some systematic uncertainties due to the linear interpolation, as is, e.g., visible for $a^{-1}=1.14 \mathrm{GeV}$ in four dimensions in Fig. 1. It has been attempted to improve the situation using a four-point spline interpolation. However, the statistical error then accumulated, while the systematic error was not strongly improved. Thus, this did not lead to a substantial reduction of the error. However, the problem is reduced more and more with finer and finer lattices at the relevant momentum regime of $\mu$, and this type of systematic error remains hence as a discretization artifact. However, it does not influence the qualitative conclusions, nor, as visible in Fig. 1, is it a substantial quantitative error.

\subsection{Scale and scheme dependence}

In Sect. 3.1 the scheme was defined by setting the mass on the right-hand side of (2) and (3) equal to the tree-level mass. In Sect. 3.2, the renormalization scale was furthermore fixed to $\mu=1.5 \mathrm{GeV}$. Both conditions are, of course, not necessary. Here, the dependence on both is studied for the case of $m=$ $1 \mathrm{GeV}$. Of course, strong deviations are expected if either $\mu$ or the masses $m$ and $m_{\mathrm{r}}$ are close to the lattice scales $1 / a$ and $N / a$. This is the reason why only the case $m=1 \mathrm{GeV}$ will be considered in more detail, and only the largest volume for which a lattice spacing $a \gtrsim(2 \mathrm{GeV})^{-1}$ is available.

The results are shown for both scale and scheme dependence in Fig. 2. The dependence of the results on the renormalization scale $\mu$ is extremely minor, and not larger than any systematic error. The situation is distinctively different for the scheme dependence. With decreasing renormalized mass $m_{\mathrm{r}}$ the propagator starts to deviate increasingly from the treelevel propagator, and the stronger the higher the dimension. Interestingly, the renormalized propagator is below the treelevel propagator, a behavior indicative of an effective mass larger than the renormalized mass. This will be confirmed in Sect. 4.

\subsection{Dependence of the renormalization constants on the volume and the cutoff}

One relevant question is the dependence of the renormalization constants on the lattice parameters. The dependence on volume is quite interesting, since if it is weak, it would allow one to determine the renormalization constants on small lattices with large statistics and, thus, very precisely. 

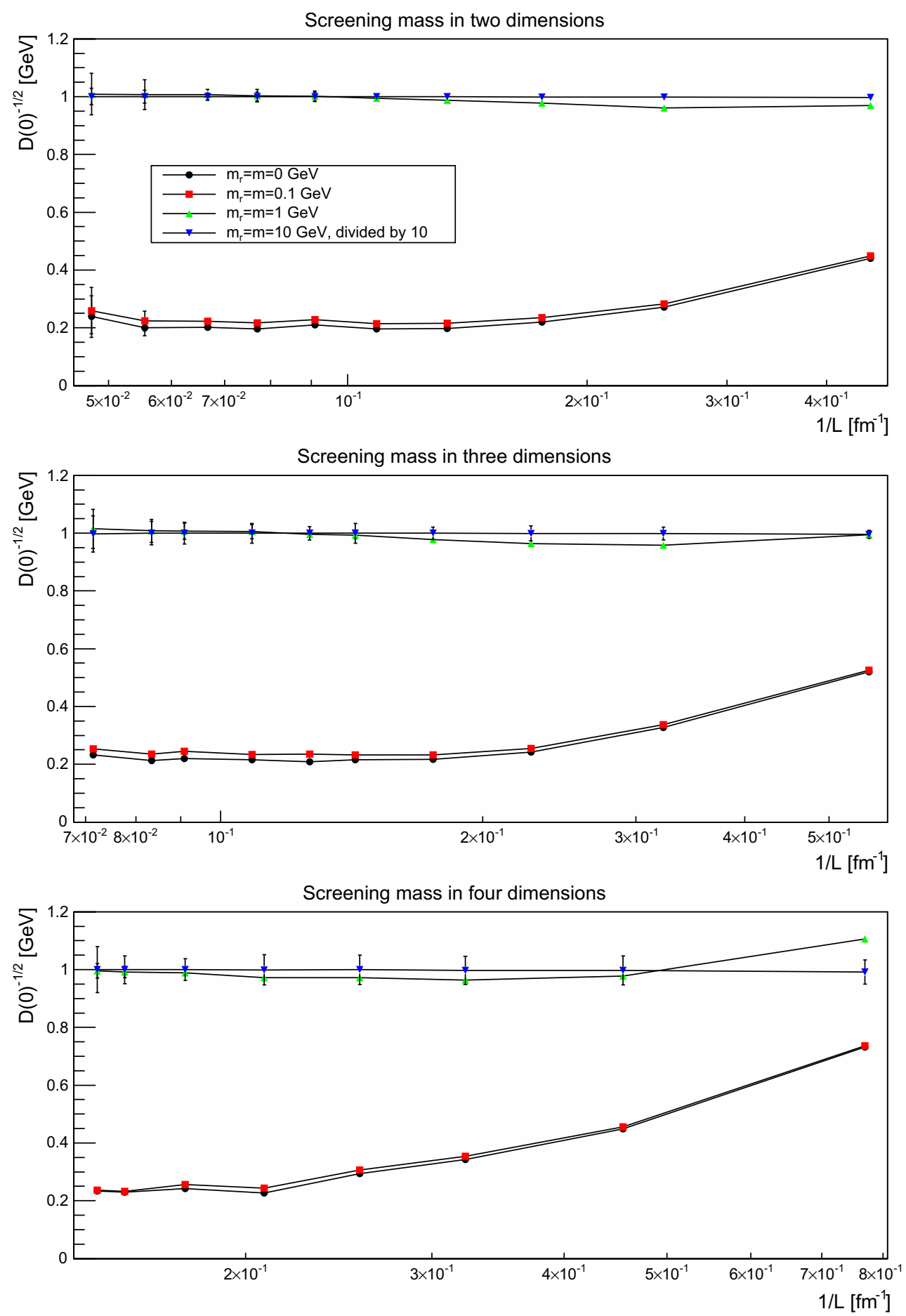

Fig. 21 The value of the screening mass $D(0)^{-1 / 2}$ as a function of lattice extent in two (top panel), three (middle panel), and four dimensions (bottom panel)

This would reduce the effect of error propagation considerably. The dependence on the cutoff is especially interesting not only as a systematic error source, but also to see whether the naive perturbative expectation [27] coincides with the actual behavior. In the following the standard scheme $m_{\mathrm{r}}=m$ with a renormalization scale $\mu=1.5 \mathrm{GeV}$ will be used.

The results for the wave-function renormalization is shown in Figs. 3, 4 and 5 for two, three, and four dimensions, respectively. All cases show a wave-function renor- 


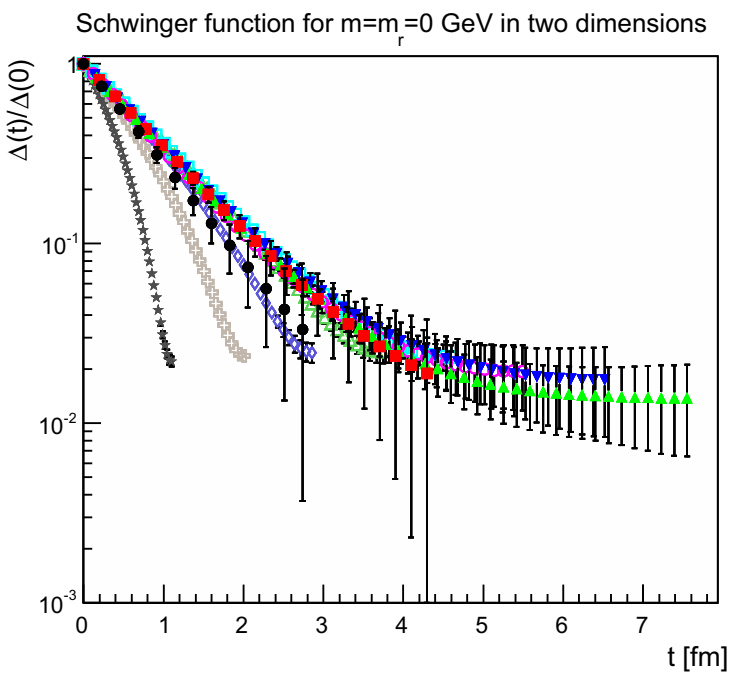

Schwinger function for $m=m=0.1 \mathrm{GeV}$ in two dimensions

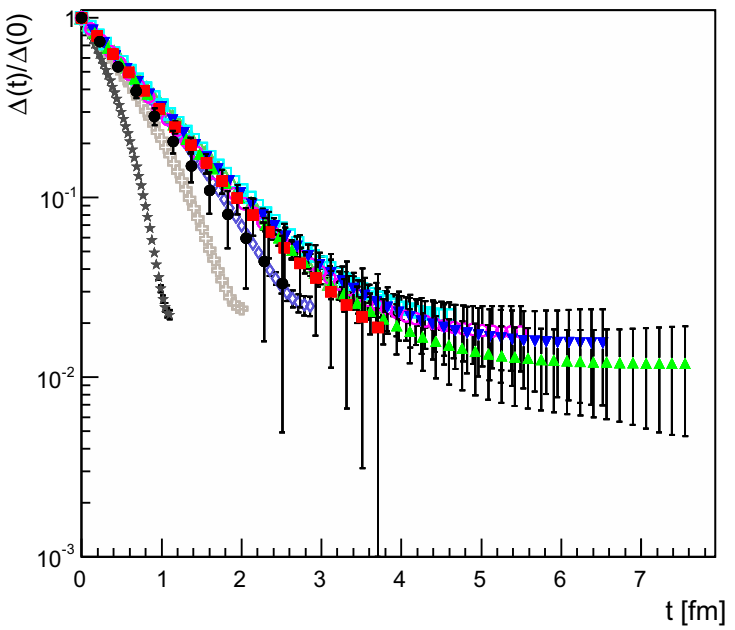

Schwinger function for $m=m=1 \mathrm{GeV}$ in two dimensions

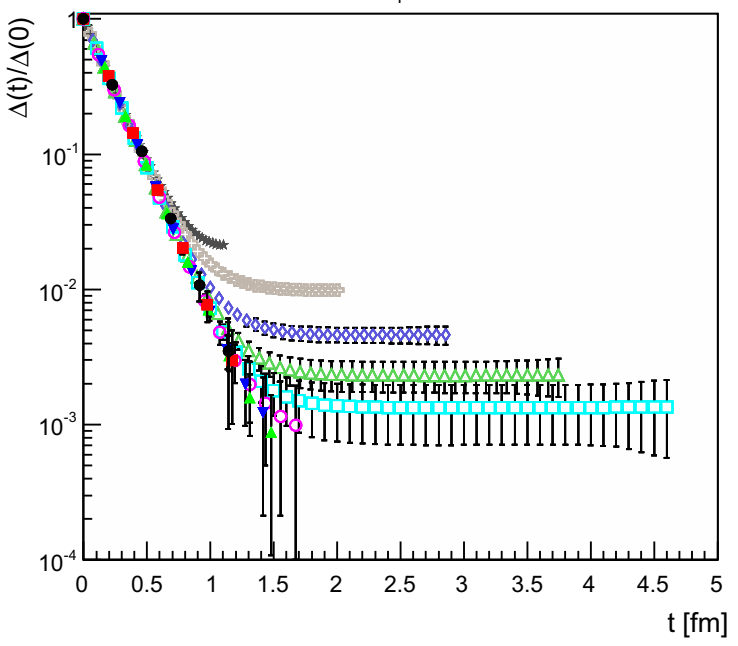

Schwinger function for $m=m_{r}=10 \mathrm{GeV}$ in two dimensions

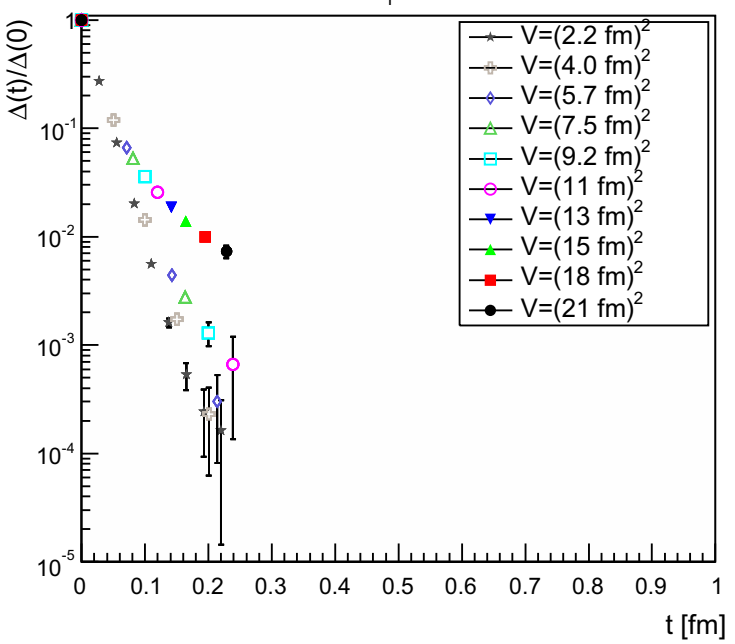

Fig. 22 The Schwinger function in two dimensions for $m=m_{\mathrm{r}}=0 \mathrm{GeV}$ (top-left panel), $m=m_{\mathrm{r}}=0.1 \mathrm{GeV}$ (top-right panel), $m=m_{\mathrm{r}}=1 \mathrm{GeV}$ (bottom-left panel), and $m=m_{\mathrm{r}}=10 \mathrm{GeV}$ (bottom-right panel). Points with a relative error larger than $100 \%$ have been omitted

malization $Z(1 / a)$ which decays with decreasing lattice spacing $a$. Volume effects are small, and especially do not affect the qualitative behavior, though there is some drift for volumes smaller than $(2-3 \mathrm{fm})^{d}$. The wave-function renormalization is essentially a continuous function of $a$ for two and three dimensions, while there is a pronounced jump in four dimensions around $a^{-1}=1-1.5 \mathrm{GeV}$, which occurs later for larger volumes. However, this effect is still rather small, and only slightly affects the quantitative behavior.

The slow evolution already suggests a logarithmic behavior. Indeed, the data can be fitted rather well using the fit form

$Z(a)=Z_{\infty}+c\left(\ln \left(\frac{\Lambda^{2}+\frac{1}{a^{2}}}{(1 \mathrm{GeV})^{2}}\right)\right)^{\epsilon}$
The resulting parameters are shown for the smallest volume in Table 1. They are also indicated as the hatched band in Figs. 3, 4 and 5.

Although the smallest volume shows some quantitative deviation from the trend of larger volumes, it allows one to reach larger cutoffs $1 / a$, and therefore more stable fits of the logarithmic tail. However, even then the values for the anomalous dimensions have large uncertainties. The remaining fit parameters show the expected behavior. The scale $\Lambda$ is of the typical scale of $1 \mathrm{GeV}$. In two and three dimensions, the renormalization constants tend to a constant (in fact essentially to 1) at infinite cutoff, showing that no wavefunction renormalization is required. This is the expected behavior from perturbation theory. In four dimensions no stable fit with a finite value of $Z_{\infty}$ was possible, which is also expected from perturbation theory [27]. 

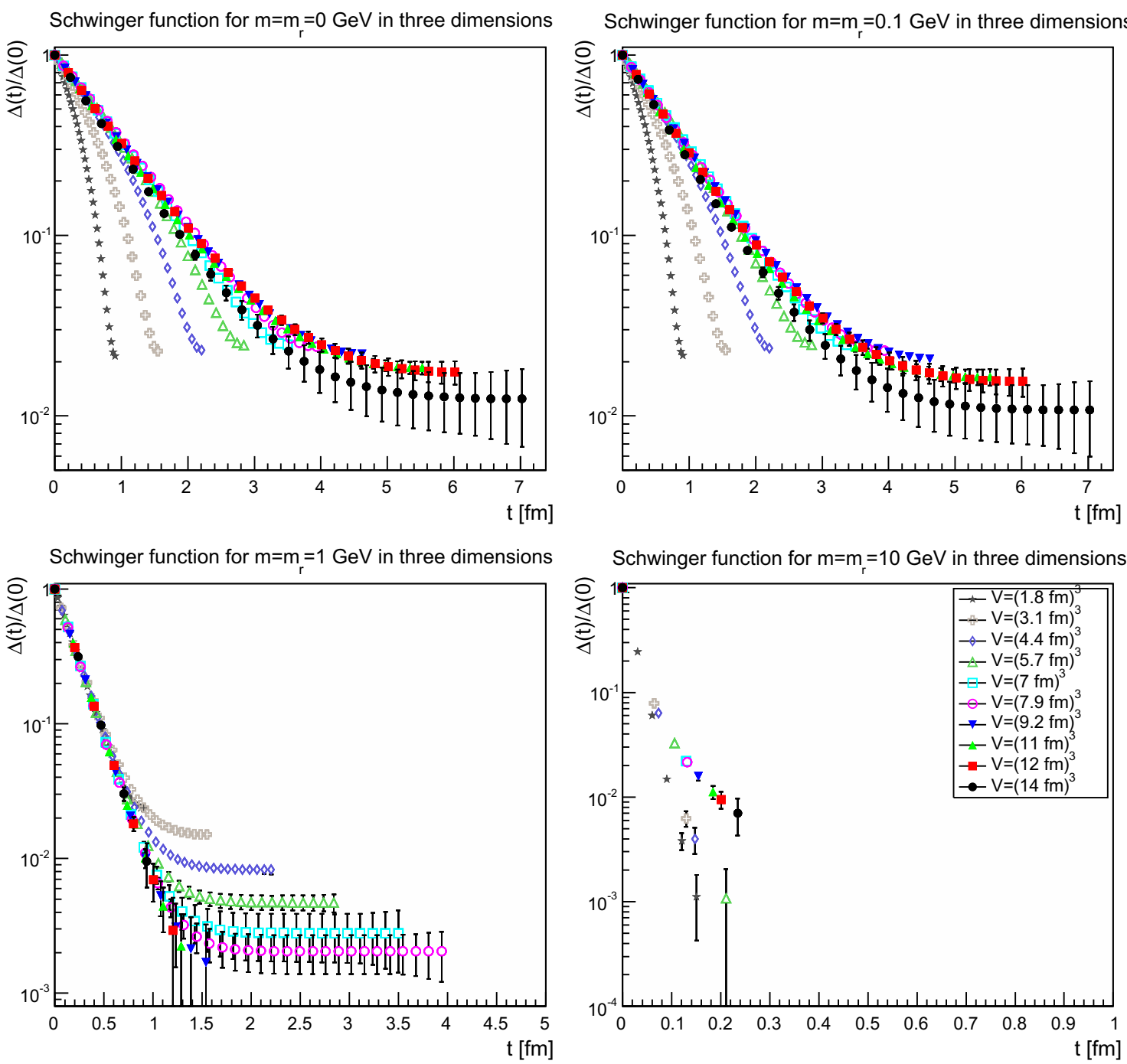

Fig. 23 The Schwinger function in three dimensions for $m=m_{\mathrm{r}}=0 \mathrm{GeV}$ (top-left panel), $m=m_{\mathrm{r}}=0.1 \mathrm{GeV}$ (top-right panel), $m=m_{\mathrm{r}}=1 \mathrm{GeV}$ (bottom-left panel), and $m=m_{\mathrm{r}}=10 \mathrm{GeV}$ (bottom-right panel). Points with a relative error larger than $100 \%$ have been omitted

Incidentally, this is already a first hint that the fundamental scalar, at least in four dimensions, is not a physical particle, due to the Öhme-Zimmermann superconvergence relation [28].

The situation for the mass renormalization constant, more precisely for $\left(\left|\delta m^{2}-m^{2}\right|\right)^{1 / 2}$, is shown in Figs. 6, 7 and 8 .

The results can be fitted rather well for the three lighter cases by the form

$\frac{\delta m^{2}(a)-m^{2}}{(1 \mathrm{GeV})^{2-\epsilon}}=-\left(m+c a^{-\delta} \ln \left(\Lambda^{2}+\frac{1}{a^{2}}\right)\right)^{\epsilon}$.

The fit parameters are listed in Table 2. As expected, the dependence on $a^{-1}$ is roughly logarithmic in two dimensions, linear in three dimensions, and quadratic in four dimensions. Again, slight modifications of the fit form work equally well, but the general trend remains the same. This form also shows that in the limit $a \rightarrow \infty$ the mass moves close to the classical mass, especially in three and four dimensions.

The situation for $m=10 \mathrm{GeV}$ is more involved. Replacing $m$ by $-m$, the fit form (5) also works in two and three dimensions, but not in four dimensions. The reason can essentially be inferred from the comparison of the three dimensionalities. In all case, there appears to be a competition between two effects. One, which pushes the mass renormalization to zero, and one which pushes it to infinity. The latter wins out earlier the higher the dimension, and is the one with the expected dependence on $a$. It appears thus reasonable that this is the actual behavior of the renormalization constant, in agreement with expectations, while the other contribution is likely a lattice artifact. Just because the divergent part grows quicker the higher the dimension this effect wins for smaller $a$ in higher dimensions, while the bending-over has not yet 

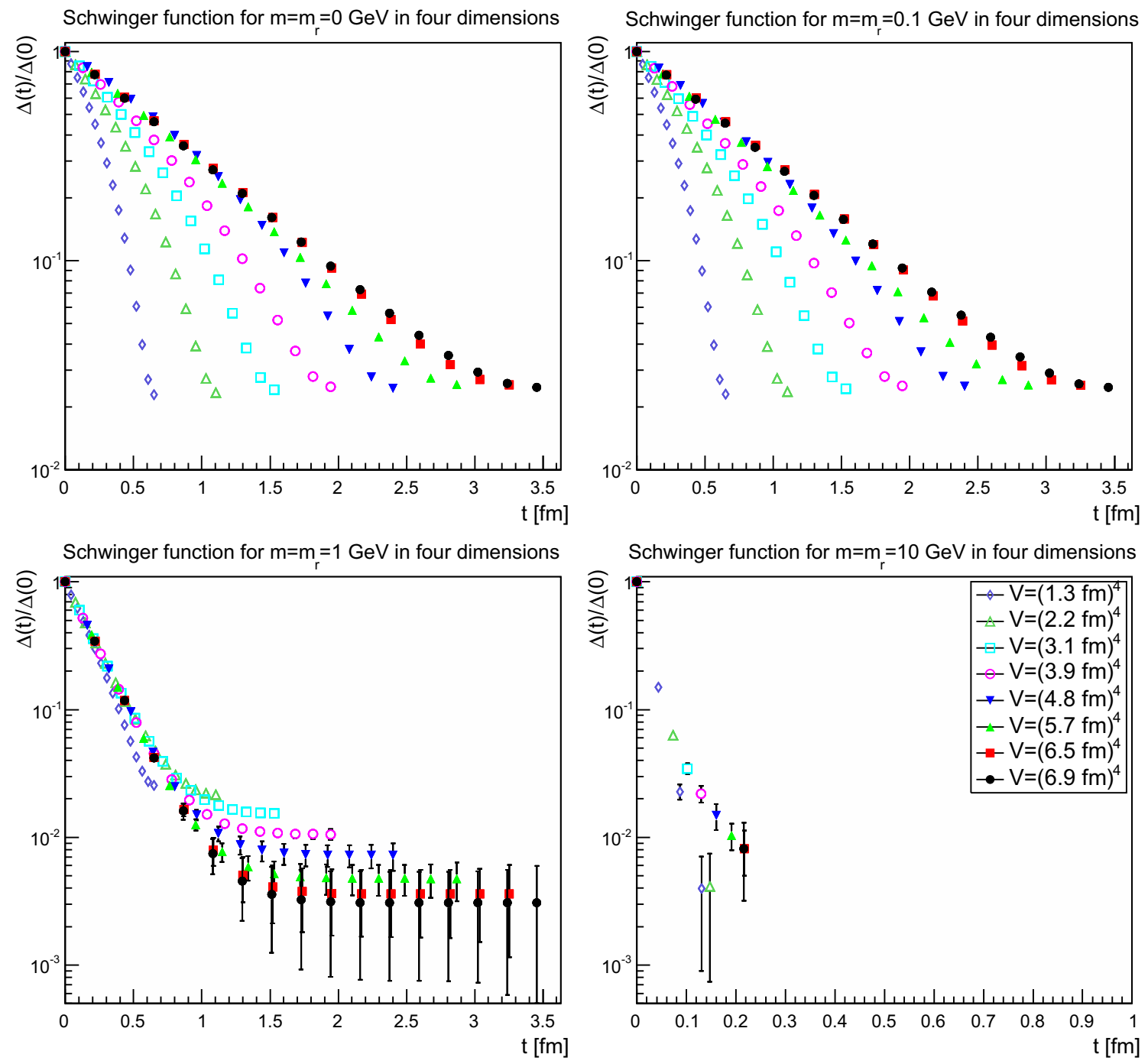

Fig. 24 The Schwinger function in four dimensions for $m=m_{\mathrm{r}}=0 \mathrm{GeV}$ (top-left panel), $m=m_{\mathrm{r}}=0.1 \mathrm{GeV}$ (top-right panel), $m=m_{\mathrm{r}}=1 \mathrm{GeV}$ (bottom-left panel), and $m=m_{\mathrm{r}}=10 \mathrm{GeV}$ (bottom-right panel). Points with a relative error larger than $100 \%$ have been omitted

been reached in two dimensions. In four dimensions, this is happening but the full behavior cannot be captured by the fit as in three dimensions. Fitting with values at large cutoff only would probably be possible, but not yet enough points are available to do this. This is also consistent with the expectation that lattice artifacts due to lattice spacings should be largest for the largest mass, and thus a similar effect for the lighter masses should be suppressed.

The comparison of the parameters in Tables 1 and 2 show for the light masses a slight dependence on the mass, as was to be expected in the present mass-dependent scheme. On the other hand, Figs. 3, 4, 5, 6, 7, and 8 show pretty clearly that once a certain minimal volume of a few $\mathrm{fm}^{d}$ has been reached, the renormalization constants are essentially volume-independent. Thus, small-volume high-statistic runs can be used to get already a reasonable result for the renormalization constants, if need would be.
With this, the renormalization of the propagators appears to be sufficiently well under control so that their analytic structure can be investigated next.

\section{Analytic structure}

\subsection{Momentum-space properties}

Since the results of the previous section, especially Fig. 1, strongly suggest that discretization artifacts are small in the following only the results for the finest lattices will be considered. In fact, checking all the lattice setups individually only shows an, almost statistically insignificant, tendency for the propagators to be a little more infrared enhanced the smaller $a$ is.

In addition to the propagator themselves the dressing functions are interesting, which will be defined as 

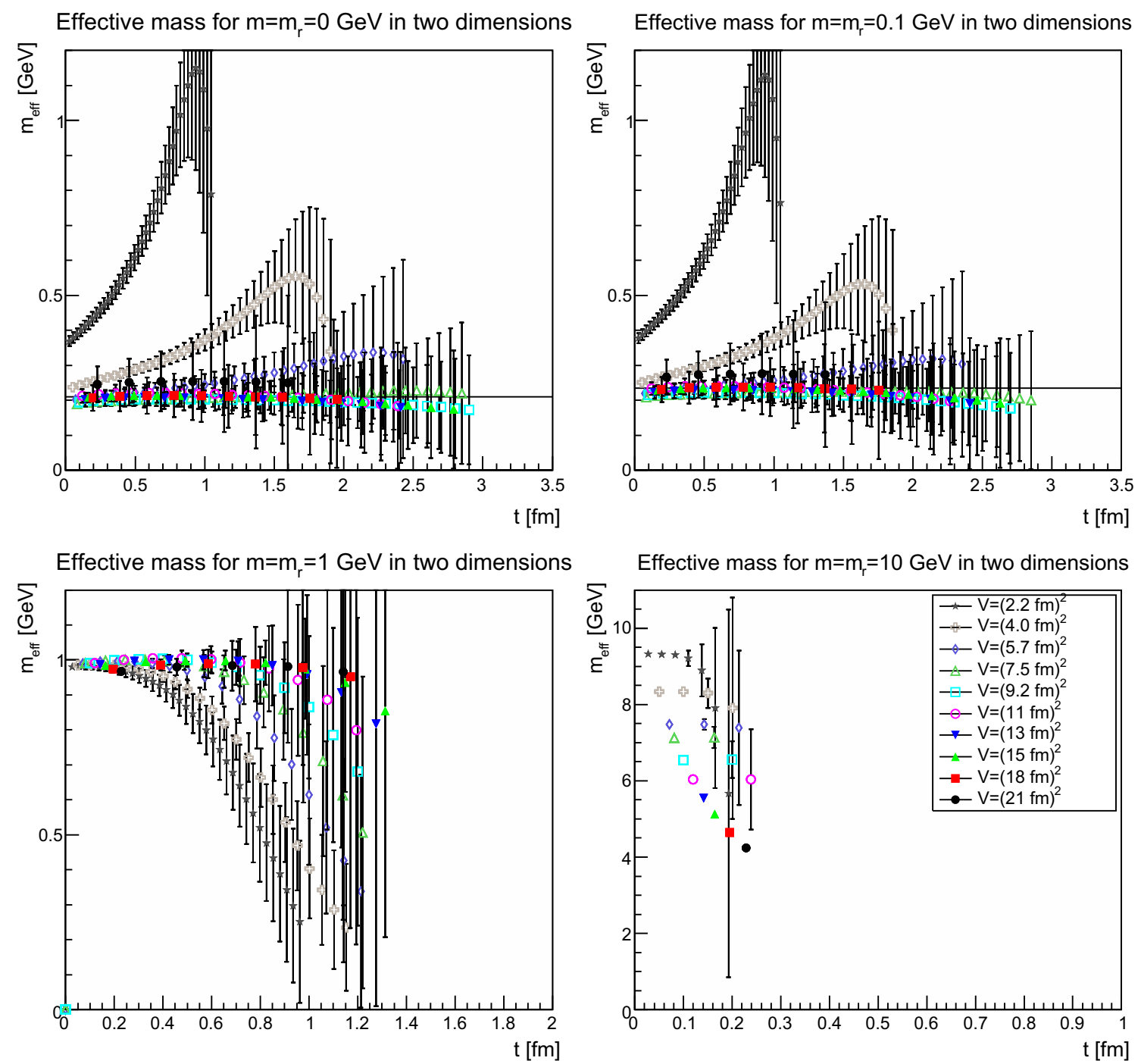

Fig. 25 The effective mass in two dimensions for $m=m_{\mathrm{r}}=0 \mathrm{GeV}$ (top-left panel), $m=m_{\mathrm{r}}=0.1 \mathrm{GeV}$ (top-right panel), $m=m_{\mathrm{r}}=$ $1 \mathrm{GeV}$ (bottom-left panel), and $m=m_{\mathrm{r}}=10 \mathrm{GeV}$ (bottom-right

panel). Points with a relative error larger than $100 \%$ have been omitted. The lines in the top-left panel and the top-right panel correspond to 210 and $235 \mathrm{MeV}$, respectively

$H\left(p^{2}\right)=\left(p^{2}+m_{\mathrm{r}}^{2}\right) D\left(p^{2}\right)$,

and which therefore describe the deviation from the corresponding tree-level propagator, and hence the influence of quantum corrections.

The results are shown for two dimensions in Figs. 9, 10, 11 and 12, for three dimensions in Figs. 13, 14, 15 and 16, and for four dimensions in Figs. 17, 18, 19 and 20. Of course, at $\mu$ all propagators have, by construction, a dressing function of 1 .

More interesting is the observation that the propagator for $m=m_{\mathrm{r}}=0 \mathrm{GeV}$ tends to a finite value in the infrared, which is also visible in the dressing functions, irrespective of the dimension. To emphasize this, the value of the propagators at zero momentum, or more precisely their screen- ing masses $D(0)^{-1 / 2}$, are shown as a function of lattice extension in Fig. 21. Note that since the propagator has not been evaluated there, this value is obtained by a linear extrapolation of the propagator at the two lowest non-zero momenta.

This is interesting as it implies that even if the renormalization condition forces the propagators to behave like a massless one at $\mu$, the actual propagator in the infrared develops a non-zero screening mass, and thus a finite value for the propagator. Hence, even though the renormalization scheme and the tree-level mass enforce a massless behavior, the dynamics induce a screening or gaping at small momenta.

The value of this additional screening mass is somewhat erratically changing from volume to volume. This indicates that a mixture of discretization and volume effects as well as 

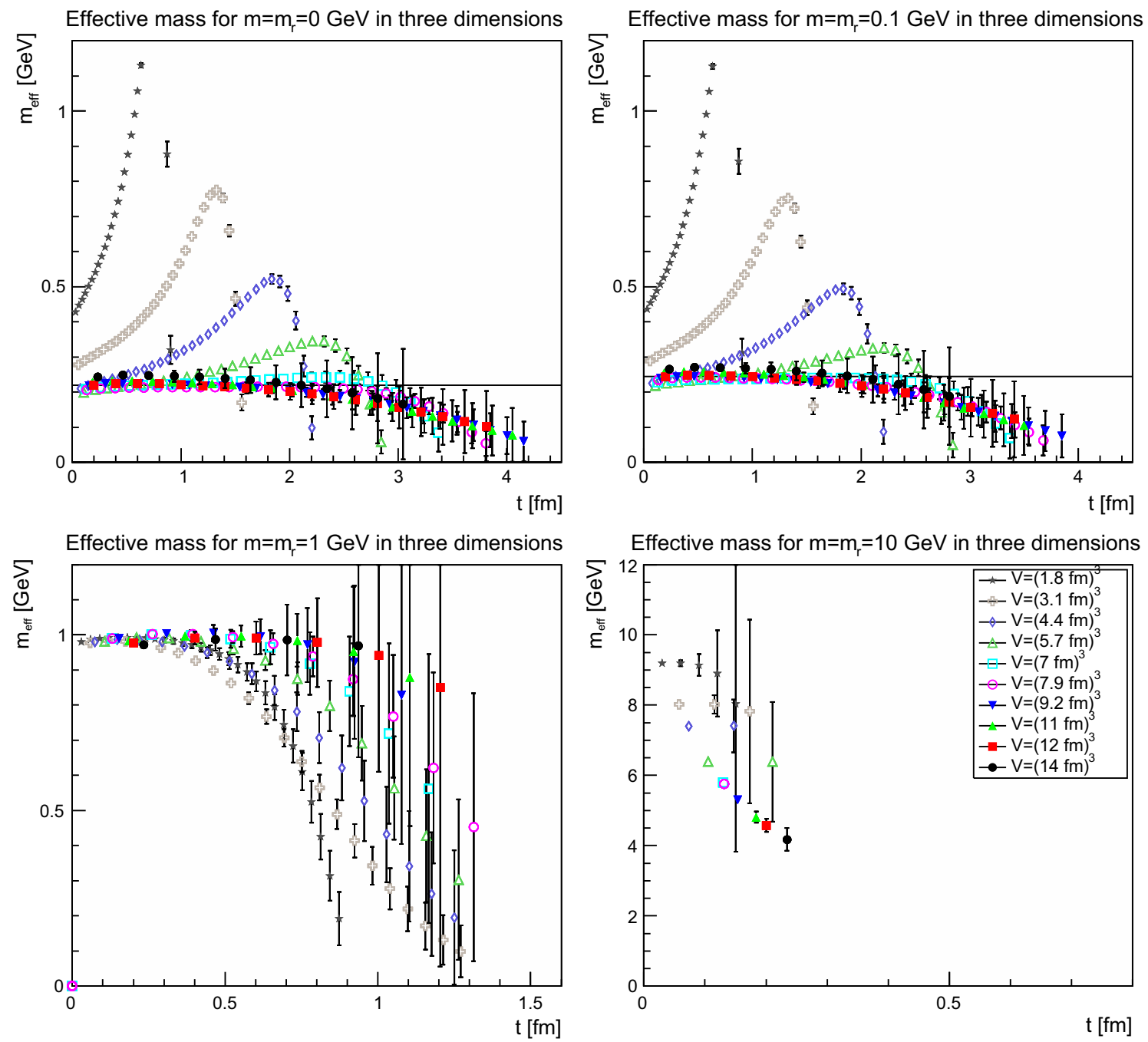

Fig. 26 The effective mass in three dimensions for $m=m_{\mathrm{r}}=0 \mathrm{GeV}$ (top-left panel), $m=m_{\mathrm{r}}=0.1 \mathrm{GeV}$ (top-right panel), $m=m_{\mathrm{r}}=$ $1 \mathrm{GeV}$ (bottom-left panel), and $m=m_{\mathrm{r}}=10 \mathrm{GeV}$ (bottom-right

panel). Points with a relative error larger than $100 \%$ have been omitted. The lines in the top-left panel and the top-right panel correspond to 220 and $245 \mathrm{MeV}$, respectively

systematic effects in the renormalization process affect the actual value.

A similar effect is also seen for larger masses. In all cases, the renormalized propagator is below the tree-level value at small momenta, though the effect becomes smaller and smaller the larger the mass. In the case of $m=m_{\mathrm{r}}=10 \mathrm{GeV}$ it is essentially gone. Indeed, the values of the propagators at small masses suggest a screening mass of the order of $200-250 \mathrm{MeV}$, which is surprisingly similar to the screening mass observed for quarks in the fundamental representation in the chiral and quenched limit $[2,4,8,11]$. This may indicate that the generation of screening masses is actual similar for bosons and fermions. However, in contrast to fermions the contribution from the screening mass seems to diminish with increasing renormalized mass, though

the additive shift in the mass may overlay this to some extent.

The behavior of the propagators at higher momenta is then following more or less the expected pattern. At high mass the propagators also start to deviate again from the tree-level one. In four dimensions, this follows from the logarithmic decay due to the renormalization effects. In lower dimensions, this is somewhat unexpected, and in contrast to the gauge propagators [3]. This is, however, likely due to the additional wave-function renormalization, which compensates partly for a self-energy contribution, and this discrepancy yields the observed effect: Due to asymptotic freedom, at large momenta all propagators in two and three dimensions tend to $D=1 /\left(Z p^{2}\right)$, yielding $H(p)=1 / Z$, rather than unity. 

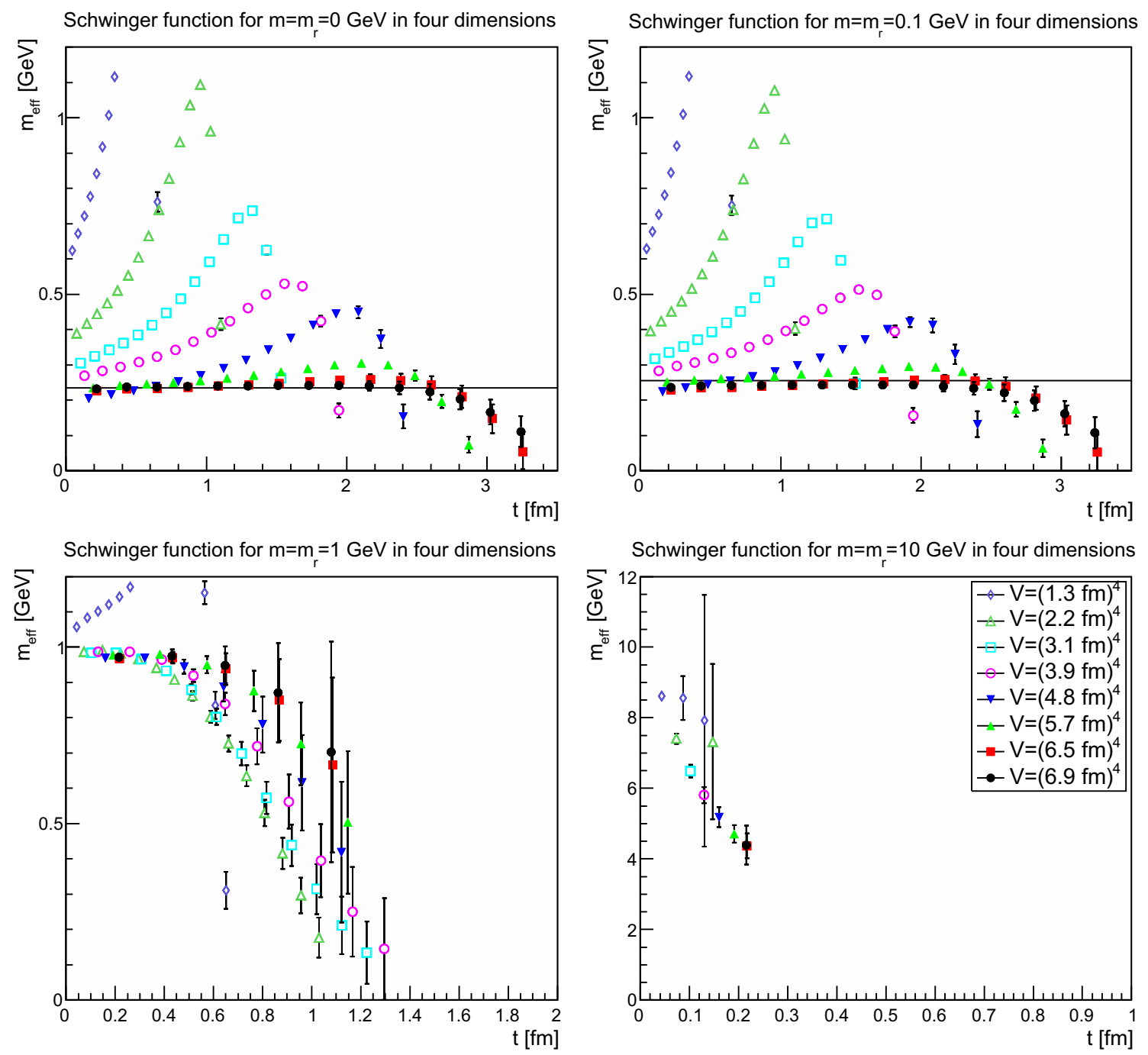

Fig. 27 The effective mass in four dimensions for $m=m_{\mathrm{r}}=0 \mathrm{GeV}$ (top-left panel), $m=m_{\mathrm{r}}=0.1 \mathrm{GeV}$ (top-right panel), $m=m_{\mathrm{r}}=$ $1 \mathrm{GeV}$ (bottom-left panel), and $m=m_{\mathrm{r}}=10 \mathrm{GeV}$ (bottom-right

panel). Points with a relative error larger than $100 \%$ have been omitted. The lines in the top-left panel and the top-right panel correspond to 235 and $255 \mathrm{MeV}$, respectively

\subsection{Schwinger function and effective mass}

The Schwinger function

$$
\begin{aligned}
\Delta(t) & =\frac{1}{\pi} \int_{0}^{\infty} \mathrm{d} p_{0} \cos \left(t p_{0}\right) D\left(p_{0}^{2}\right) \\
& =\frac{1}{a \pi} \frac{1}{N_{\mathrm{t}}} \sum_{P_{0}=0}^{N_{\mathrm{t}}-1} \cos \left(\frac{2 \pi t P_{0}}{N_{\mathrm{t}}}\right) D\left(P_{0}^{2}\right),
\end{aligned}
$$

essentially the temporal correlator, is obtained from the renormalized propagator. The calculation is straightforward in principle, though requires obtaining the removed value at zero momentum. As above, this is obtained by a linear extrapolation of the propagator at the two lowest momenta. Especially for the larger masses this is relatively accurate, but induces some systematic error for the smaller masses on

small physical volumes. However, this only adds a constant to the Schwinger function, which yields a negative contribution to the effective mass. This offset vanishes as a function of the physical volume.

These correlation functions have a very simple behavior, a sum of exponentials, for gauge-invariant Euclidean correlation functions on any finite lattice [29]. The situation is quite different for gauge-dependent correlation functions [2,3,12]: There are no general constraints.

From the Schwinger function the effective (timedependent) mass

$m_{\mathrm{eff}}=-\ln \frac{\Delta(t+a)}{\Delta(t)}$,

can be derived, which in the case of a simple exponential decay coincides with the usual mass. On a finite lattice, for 

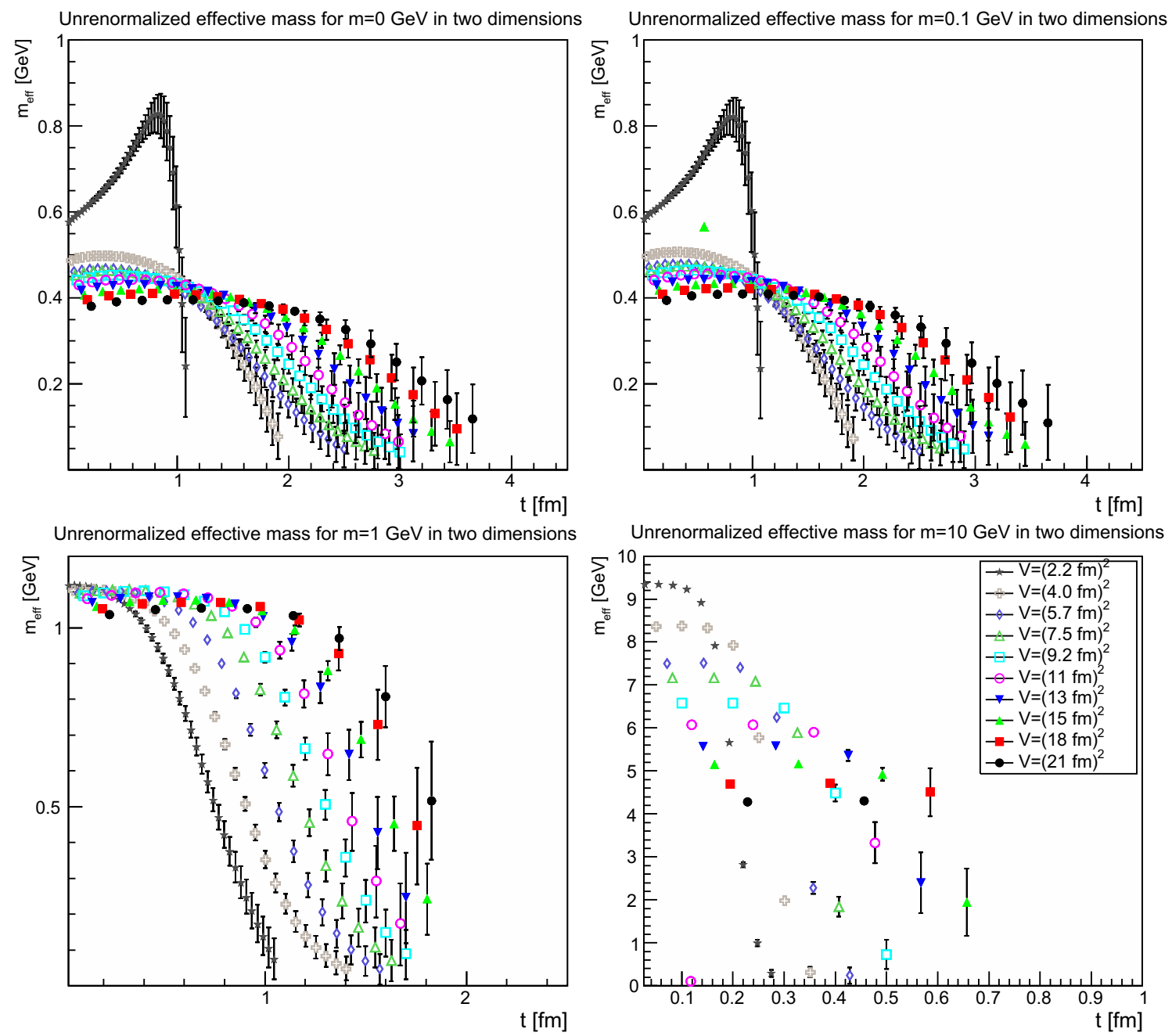

Fig. 28 The unrenormalized effective mass in two dimensions for $m=0 \mathrm{GeV}$ (top-left panel), $m=0.1 \mathrm{GeV}$ (top-right panel), $m=1 \mathrm{GeV}$ (bottom-left panel), and $m=10 \mathrm{GeV}$ (bottom-right panel). Points with a relative error larger than $100 \%$ have been omitted

any physical particle with a positive spectral function this effective mass is a monotonously decreasing function for $t \leq L / 2$, and corresponds to the effective mass at a given time. Eventually, at sufficiently long time, it is just the mass of the ground state.

If the effective mass is non-monotonous decreasing, the spectral function has necessarily negative contributions. Therefore it does not describe a physical particle.

The Schwinger functions and the corresponding effective masses are shown in Figs. 22, 23 and 24 and 25, 26 and 27, respectively.

The Schwinger function themselves show a number of interesting features. First of all, the results are quite similar, independent of dimensions. Secondly, finite-volume effects are visible, especially for small physical volumes. Since the physical volumes become smaller with increasing dimension, this effect is emphasized with increasing dimension. This effect is also much stronger for the smaller renormalized masses. In the case of $10 \mathrm{GeV}$, the drop is so sharp that for almost no relevant time distance a reasonable statement can be made before the signal drowns in noise.

The third observation is that the Schwinger function is positive for all times. This is in stark contrast to the gluon propagator [3] and the (quenched) adjoint quark propagator [13], which show such violations of positivity on a time scale of order $1 \mathrm{fm}$. Most interesting would be a comparison to the fundamental quark propagator, as both are in the same representation and both are affected in the same way by the Wilson confinement criterion. But here the situation is yet undecided [12], though the indications also suggest a violation of positivity. It is also unclear whether a violation of positivity is seen for the unquenched fundamental scalar [19] for QCD-like regions of the phase diagram. For Higgslike regions of the phase diagram there is no indication of a positivity violation. 

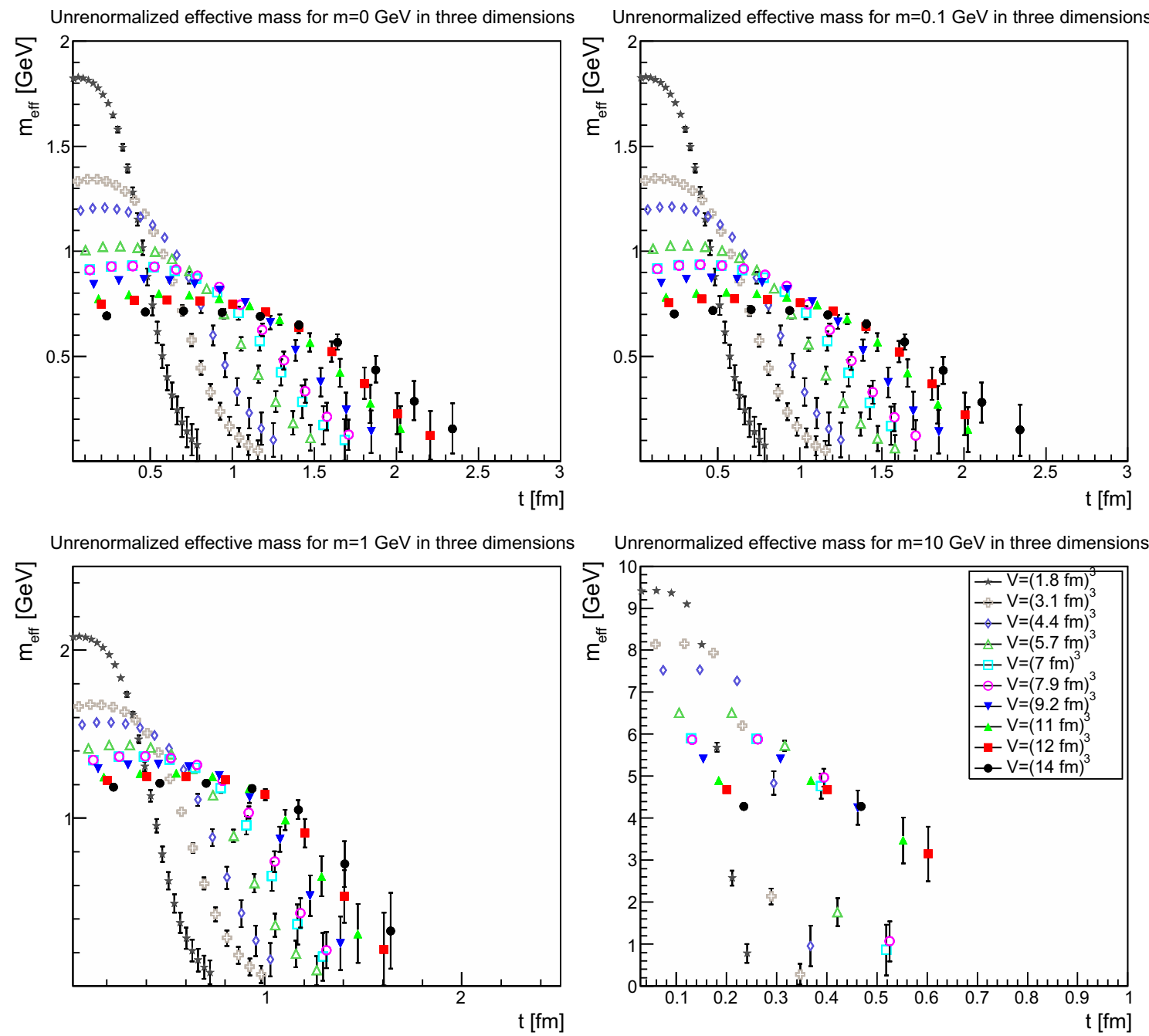

Fig. 29 The unrenormalized effective mass in three dimensions for $m=0 \mathrm{GeV}$ (top-left panel), $m=0.1 \mathrm{GeV}$ (top-right panel), $m=1 \mathrm{GeV}$ (bottom-left panel), and $m=10 \mathrm{GeV}$ (bottom-right panel). Points with a relative error larger than $100 \%$ have been omitted

Thus, the absence of an explicit positivity violation in the quenched case is an intriguing result, as it is so far the first correlator to show this behavior in a sufficiently reliable way. However, whether this has something to do with the absence of string-breaking in the quenched case is at this point at best speculation.

Though there is no such explicit violation of positivity, this by no means implies that the corresponding spectral density is positive. This is already visible by eye for small volumes, where the bending of the Schwinger function is clearly wrong for the two lightest masses. However, with increasing physical volume this type of wrong bending is reduced. There is also no obvious sign of it at the larger mass of $1 \mathrm{GeV}$.

Turning the view to the effective masses in Figs. 25-27 provides some more intriguing results. First of all, again the dependence is very similar in all dimensions, and thus no indication of a difference between dynamical and geometrical confinement.
Secondly, there is a very strong volume dependence. For small physical volumes, the effective mass bends upwards, which is not compatible with the properties of a physical particle. This behavior is substantially reduced the larger the volume up to the point where the behavior looks like that of a physical particle, with an essentially correctly curved effective mass. In fact, any deviation from the physical curvature becomes so small that statistical and systematic errors are too large to see if it remains. Such a behavior is not observed for either the gluon nor the adjoint quark propagator $[3,13]$ and was thus not expected.

The third interesting observation is that for the lightest two masses the so obtained effective mass is about $200-300 \mathrm{MeV}$, despite the renormalization condition attempts to force them to much smaller values. For the renormalized mass of $1 \mathrm{GeV}$, there is a small deviation downwards of the effective mass, before a strong suppression sets in. For the largest renormalized mass of $10 \mathrm{GeV}$, there are too few points to make any 

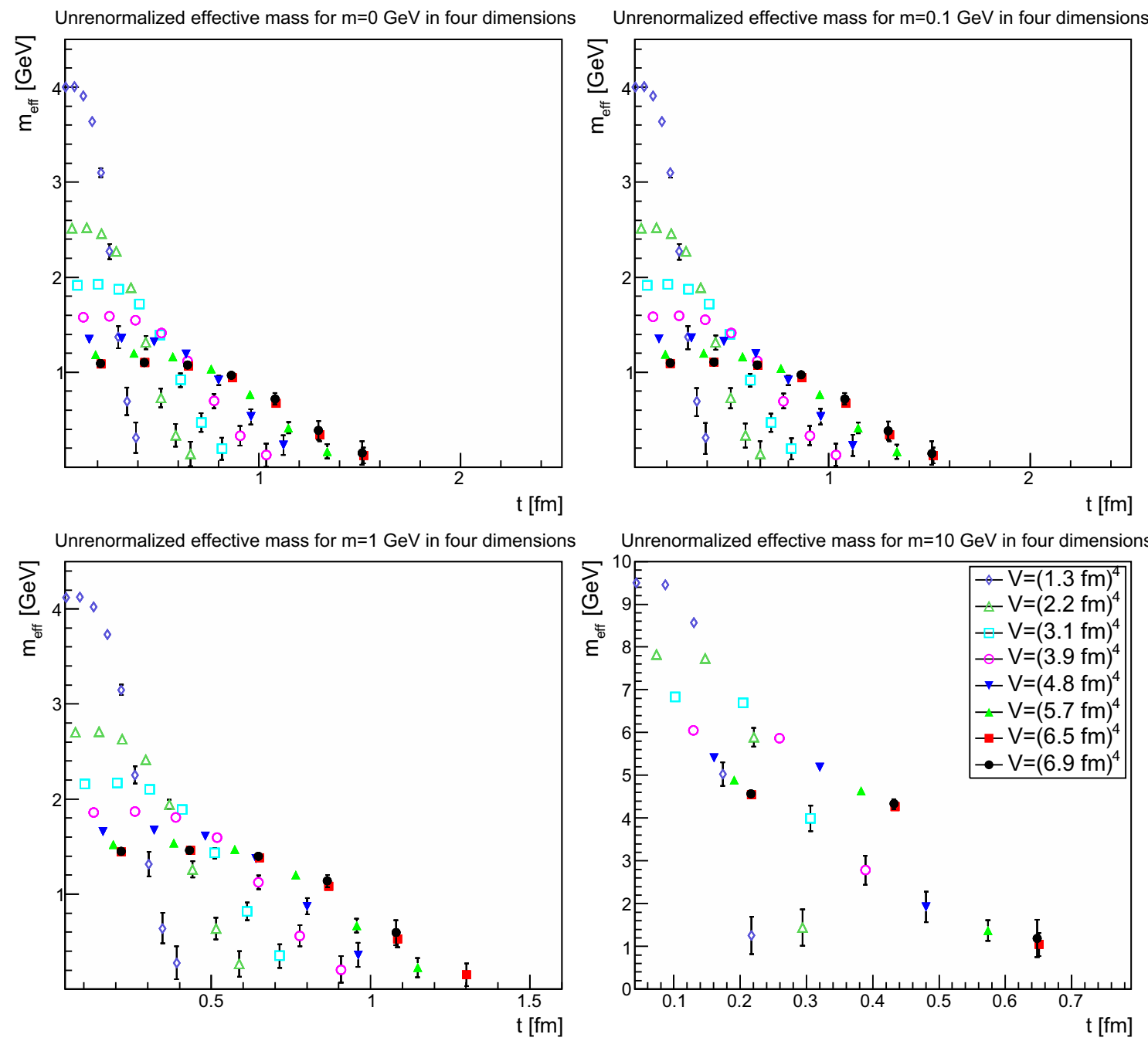

Fig. 30 The unrenormalized effective mass in four dimensions for $m=0 \mathrm{GeV}$ (top-left panel), $m=0.1 \mathrm{GeV}$ (top-right panel), $m=1 \mathrm{GeV}$ (bottom-left panel), and $m=10 \mathrm{GeV}$ (bottom-right panel). Points with a relative error larger than $100 \%$ have been omitted

firm statement, but the reliable points are at large volumes clearly incompatible with an effective mass as large as the renormalized mass.

An investigation of the lattice spacing dependence shows that for the three lightest masses the lattice spacing has essentially no impact, but the effective mass for the largest mass substantially increases. Thus, this undershooting is most probably one of the expected discretization artifacts for such a large renormalized mass.

These observations, especially for the lightest mass, are very good in line with the observations for the propagators in momentum space in Sect. 4.1, which also showed a stronger screening for the lighter two masses than just from the influence of the renormalized mass. Especially, it fits well with the results for the screening mass in Fig. 21. This suggests that there is an intrinsic mass scale in the infrared.

\subsection{Renormalization effects on the effective mass}

To understand whether the mass scale, as well as the other properties of the effective mass, are something which could be interpreted as a feature of physics, it is necessary to understand the dependence on the necessary renormalization. Of course, even if this would signal any kind of interesting feature, this would still require one to check the gauge dependence of the results, which is beyond the scope of the present work.

If the effective mass should have a physical property it should be renormalization-independent, and therefore already be a feature of the unrenormalized mass function. This possibility is investigated in Figs. ${ }^{3} 28,29$ and 30. The

\footnotetext{
3 The fact that the errors are substantially smaller at long times than for the renormalized propagator is due to the error propagation from the mass renormalization, even though these errors are just at the
} 

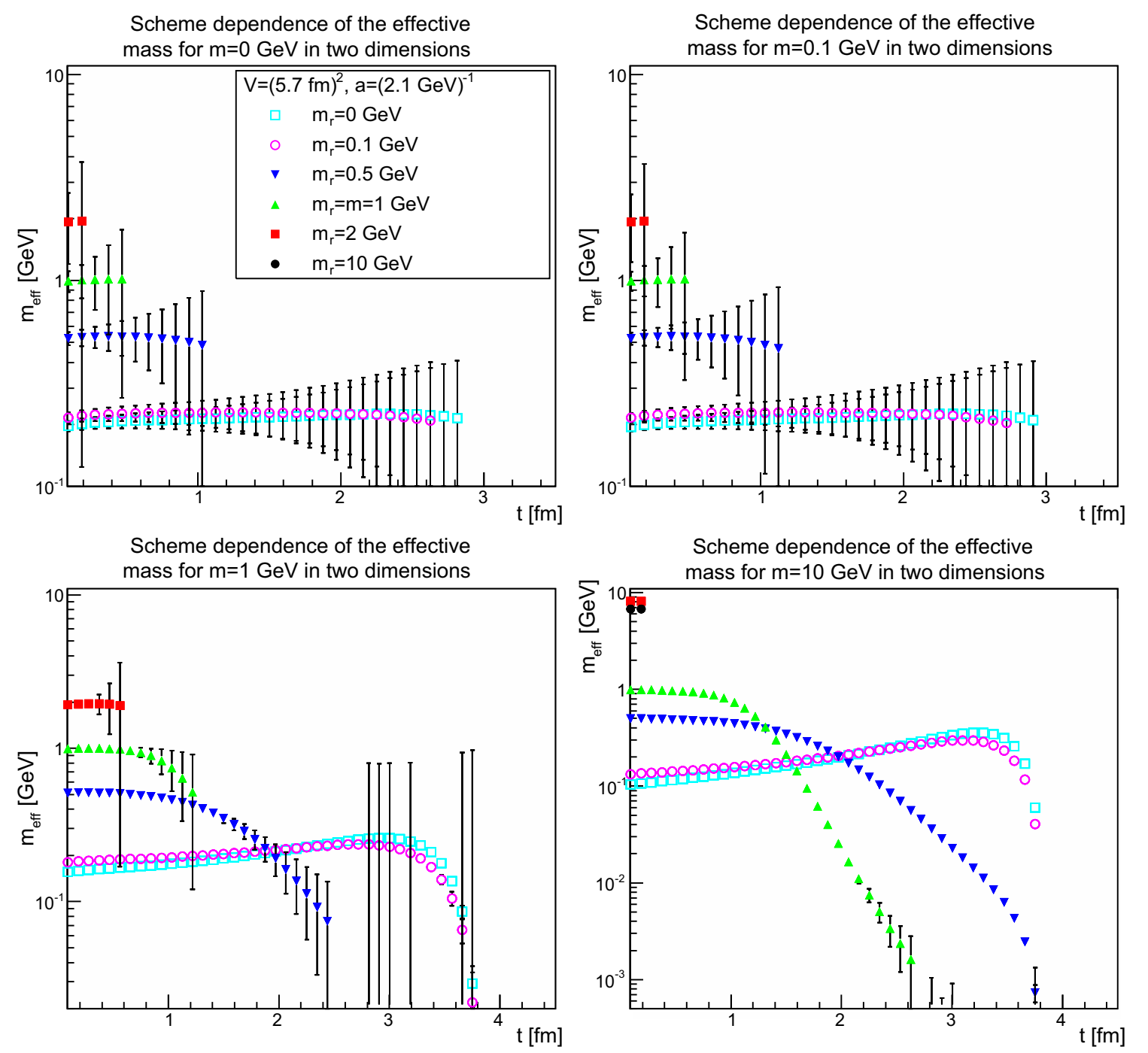

Fig. 31 The scheme dependence of the effective mass in two dimensions for $m=0 \mathrm{GeV}$ (top-left panel), $m=0.1 \mathrm{GeV}$ (top-right panel), $m=1 \mathrm{GeV}$ (bottom-left panel), and $m=10 \mathrm{GeV}$ (bottom-right panel). Points with a relative error larger than $100 \%$ have been omitted

results show a rather clear trend. First of all, in all cases it is now visible that the effective mass has an unphysical bending. Also, there is a substantial drift with the physical volume, which lets the mass drift toward zero. Both facts already suggest that the effective mass is not a physical quantity.

This becomes even more evident when considering the scheme dependence of the effective mass shown in Figs. 31, 32 and 33. It is quite visible that the effective mass strongly drifts as a function of the renormalized mass. There is a particular interesting observation to be made.

Footnote 3 continued

few percent level. The errors of the wave-function renormalization also contribute, but they are much smaller.
The effective mass is found to be of the order of the renormalized mass, as long as the renormalized mass is larger than about $200 \mathrm{MeV}$. Below this, the effective mass is not lowered further. This suggests again an intrinsic scale, independent of the bare or renormalized mass, of about this size. That the tree-level mass plays no substantial role in this is probably the most unexpected feature. It suggests that the long-distance behavior is only dominated by the interaction, not by the particle properties, as even a classically heavy particle is at most impeded in its movement by the mass scale introduced by the interaction.

This scale is not a real mass scale, as the unphysical bending of the effective mass shows. In fact, this bending increases the lower the renormalized mass and the lower the bare mass. At sufficiently large renormalized mass, the effective mass shows no sign of an unphysical bending, while it is very 

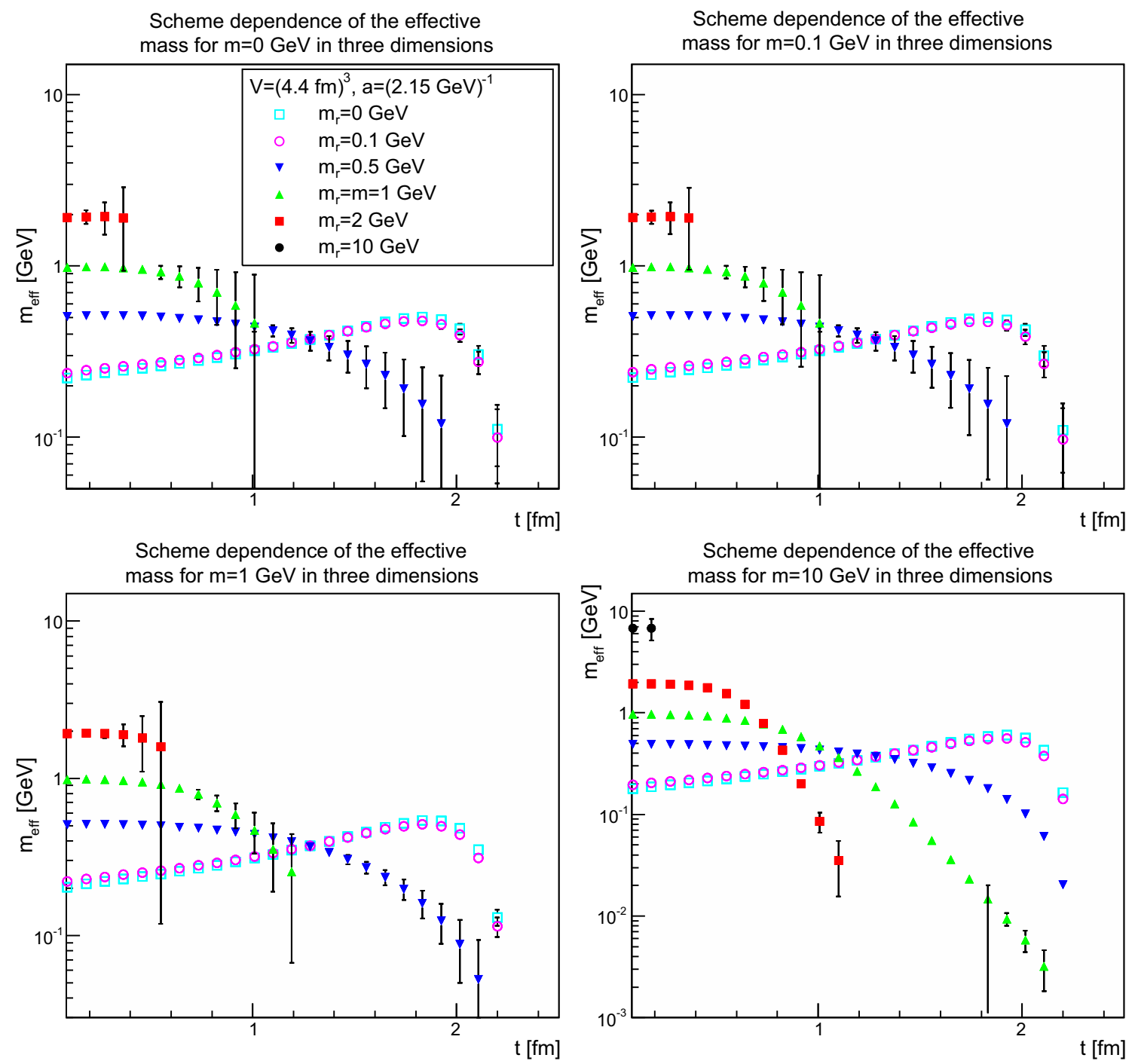

Fig. 32 The scheme dependence of the effective mass in three dimensions for $m=0 \mathrm{GeV}$ (top-left panel), $m=0.1 \mathrm{GeV}$ (top-right panel), $m=1 \mathrm{GeV}$ (bottom-left panel), and $m=10 \mathrm{GeV}$ (bottom-right panel). Points with a relative error larger than $100 \%$ have been omitted

strong, even for large bare masses, at small renormalized mass. This unphysical bending is reduced when increasing the physical volume, as is seen in Figs. 25, 26 and 27, where the same unphysical bending is observed for small renormalized masses and small physical volumes.

The renormalized mass therefore seems to screen the unphysical properties of the correlator. The systematic uncertainty due to the extrapolation of the propagator to zero momentum cannot be the source of this problem, as this only decreases, but not increases, the correlator. The best explanation is the presence of a volume-dependent effective mass, which, however, only hampers long distance propagation. Therefore, the mass increases when going to long times. This is very different from the behavior of any finite-volume artifact for physical particles [30,31].
Speculating, the results are consistent with an effective mass, and correlator, which becomes more and more in line with a physical correlator with a single, simple mass pole in the infinite-volume limit. However, the particle is still gaugedependent, and the mass scheme-dependent, and therefore neither is observable. Furthermore, there is a lower limit to this potential pole mass, below which no renormalization scheme can lower the mass.

It would be quite interesting to check for such a behavior also in the full case, especially in Higgs-like physics, along the lines of [19]. In the context of the FröhlichMorchio-Strocchi mechanism [19,32-34] any such lower mass bound for the pole mass could have rather interesting implications for the validity of perturbative spectrum calculations. 

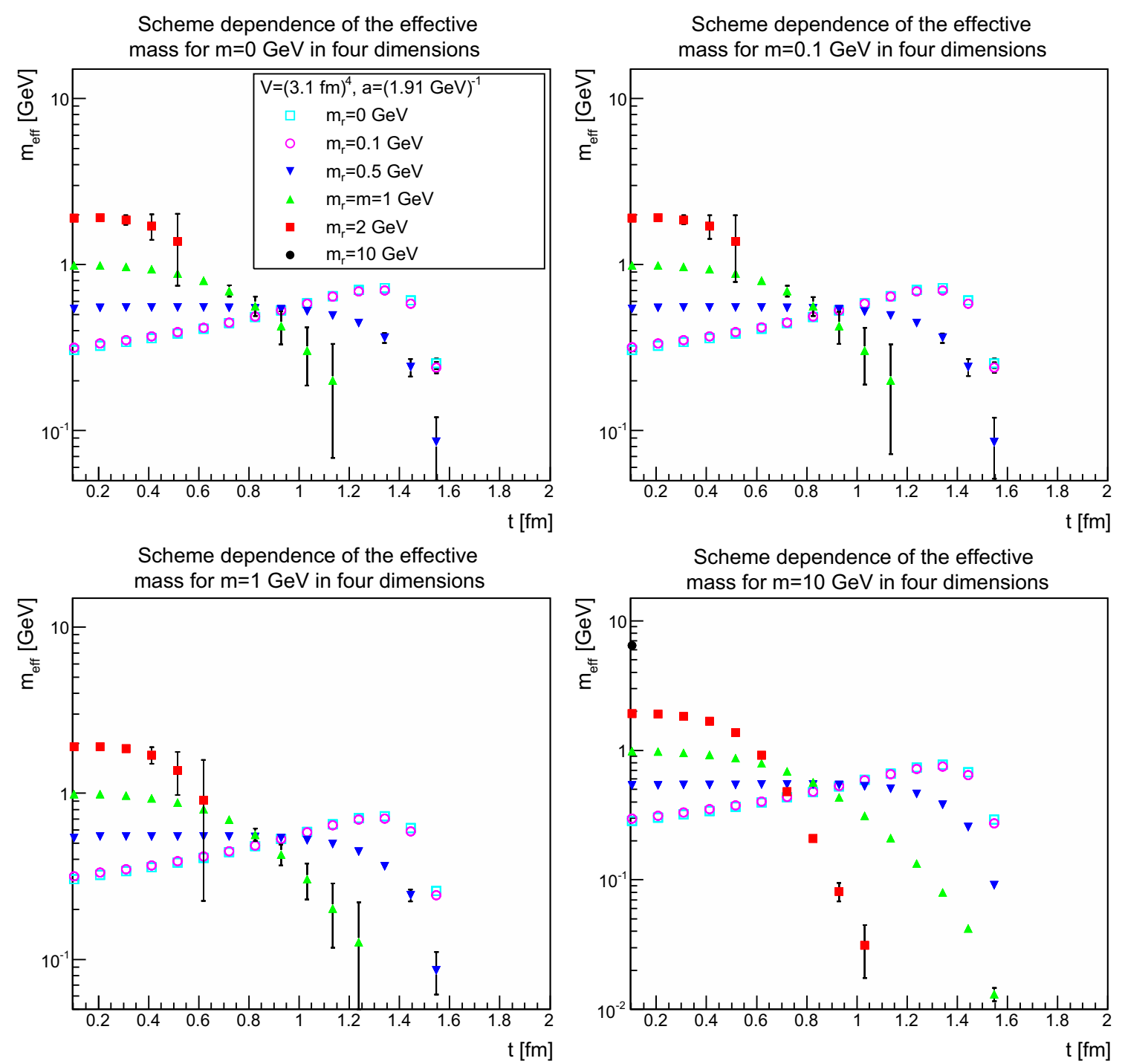

Fig. 33 The scheme dependence of the effective mass in four dimensions for $m=0 \mathrm{GeV}$ (top-left panel), $m=0.1 \mathrm{GeV}$ (top-right panel), $m=1 \mathrm{GeV}$ (bottom-left panel), and $m=10 \mathrm{GeV}$ (bottom-right panel). Points with a relative error larger than $100 \%$ have been omitted

\section{Conclusions}

Summarizing, a systematic study of the quenched, fundamental scalar propagator has been performed in two, three, and four dimensions. The renormalization properties coincide with those of perturbation theory, as anticipated in an asymptotically free theory.

The analytical structure, however, is less trivial to interpret. It appears that the propagator behaves more and more like that of an ordinary particle with a single mass pole the larger the physical volume is, though at any finite volume it retains unphysical contributions. Furthermore, the pole mass, for sufficiently large renormalized mass, coincides with the renormalized mass, provided the lattice spacing is sufficiently small to resolve the corresponding scale. However, it appears not to be possible to lower the pole mass by the renormalization condition below a certain threshold, independent of the bare mass. This threshold is, rather independent of the dimensionality, about $200-250 \mathrm{MeV}$. Thus, the propagator seems to have an intrinsic non-perturbative mass scale. This may be due to a similar effect as the constituent (screening) mass of quarks [12]. A direct comparison is, due to the additive mass renormalization, not trivial.

Still, even if true, this does not make the pole mass of the fundamental scalar any more physical, as a change in renormalization scheme can shift it to larger masses. It is still a tantalizing question whether a similar intrinsic mass scale exists in the unquenched case, where this has not yet been investigated systematically. It would also be very interesting to see how the situation in the quenched adjoint case is [15, 26,35], and whether any differences are present, a question which is currently under investigation $[18,36]$. 
Acknowledgments This work was supported by the DFG under Grant Numbers MA 3935/5-1, MA-3935/8-1 (Heisenberg program) and the FWF under Grant Number M1099-N16. Simulations were performed on the HPC clusters at the Universities of Jena and Graz. The author is grateful to the HPC teams for the very good performance of the clusters. The ROOT framework [37] has been used in this project.

Open Access This article is distributed under the terms of the Creative Commons Attribution 4.0 International License (http://creativecomm ons.org/licenses/by/4.0/), which permits unrestricted use, distribution, and reproduction in any medium, provided you give appropriate credit to the original author(s) and the source, provide a link to the Creative Commons license, and indicate if changes were made.

Funded by SCOAP ${ }^{3}$.

\section{A Lattice setups}

The various lattice setups are listed in Table 3. The determination of the lattice spacings has been performed as in [22].

Table 3 Number and parameters of the configurations used, ordered by dimension, lattice spacing, and physical volume. In all cases $2(10 \mathrm{~N}+$ $100(d-1))$ thermalization sweeps and $2(N+10(d-1))$ decorrelation sweeps of mixed updates [25] have been performed, and autocorrelation times of local observables have been monitored to be at or below one sweep. The number of configurations were selected such as to have a reasonable small statistical error for the renormalization constants determined in Sect. 3. The value $m_{0}$ denotes the value of the mass parameter in (1) to yield a tree-level mass of $1 \mathrm{GeV}$. The other tree-level masses are obtained by multiplying or dividing this number by 10 , or setting it to zero for tree-level mass zero

\begin{tabular}{llllllll}
\hline$d$ & $N$ & $\beta$ & $a(\mathrm{fm})$ & $a^{-1}(\mathrm{GeV})$ & $L(\mathrm{fm})$ & $m_{0}$ & Config. \\
\hline 2 & 92 & 6.23 & 0.228 & 0.863 & 21 & 1.159 & 2146 \\
2 & 80 & 6.40 & 0.225 & 0.875 & 18 & 1.143 & 3957 \\
2 & 58 & 6.45 & 0.224 & 0.879 & 13 & 1.138 & 3386 \\
2 & 18 & 6.55 & 0.222 & 0.886 & 4.0 & 1.129 & 3661 \\
2 & 34 & 6.64 & 0.221 & 0.893 & 7.5 & 1.120 & 2970 \\
2 & 68 & 6.64 & 0.221 & 0.893 & 15 & 1.120 & 3456 \\
2 & 10 & 6.68 & 0.220 & 0.895 & 2.2 & 1.117 & 2192 \\
2 & 50 & 6.68 & 0.220 & 0.895 & 11 & 1.117 & 3299 \\
2 & 26 & 6.72 & 0.219 & 0.898 & 5.7 & 1.113 & 3410 \\
2 & 42 & 6.73 & 0.219 & 0.900 & 9.2 & 1.112 & 3370 \\
2 & 92 & 8.33 & 0.196 & 1.01 & 18 & 0.9933 & 1958 \\
2 & 68 & 8.70 & 0.191 & 1.03 & 13 & 0.9708 & 3456 \\
2 & 58 & 8.83 & 0.190 & 1.04 & 11 & 0.9632 & 3386 \\
2 & 80 & 9.03 & 0.188 & 1.05 & 15 & 0.9519 & 3597 \\
2 & 50 & 9.36 & 0.184 & 1.07 & 9.2 & 0.9341 & 3174 \\
2 & 42 & 9.91 & 0.179 & 1.10 & 7.5 & 0.9066 & 3433 \\
2 & 34 & 11.1 & 0.168 & 1.17 & 5.7 & 0.8543 & 2950 \\
2 & 92 & 11.7 & 0.164 & 1.20 & 15 & 0.8312 & 4994 \\
2 & 80 & 11.8 & 0.163 & 1.21 & 13 & 0.8275 & 3498 \\
2 & 68 & 11.9 & 0.162 & 1.21 & 11 & 0.8239 & 3456 \\
\hline
\end{tabular}

Table 3 continued

\begin{tabular}{|c|c|c|c|c|c|c|c|}
\hline$d$ & $N$ & $\beta$ & $a(\mathrm{fm})$ & $a^{-1}(\mathrm{GeV})$ & $L(\mathrm{fm})$ & $m_{0}$ & Config. \\
\hline 2 & 58 & 12.4 & 0.159 & 1.24 & 9.2 & 0.8065 & 3304 \\
\hline 2 & 26 & 13.1 & 0.154 & 1.28 & 4.0 & 0.7838 & 3410 \\
\hline 2 & 50 & 13.8 & 0.150 & 1.31 & 7.5 & 0.7629 & 3174 \\
\hline 2 & 92 & 15.5 & 0.142 & 1.39 & 13 & 0.7185 & 2146 \\
\hline 2 & 80 & 16.3 & 0.138 & 1.43 & 11 & 0.7001 & 2279 \\
\hline 2 & 42 & 16.8 & 0.136 & 1.45 & 5.7 & 0.6893 & 3350 \\
\hline 2 & 68 & 16.9 & 0.135 & 1.46 & 9.2 & 0.6872 & 3420 \\
\hline 2 & 58 & 18.4 & 0.130 & 1.52 & 7.5 & 0.6578 & 3304 \\
\hline 2 & 18 & 20.6 & 0.122 & 1.61 & 2.2 & 0.6208 & 3660 \\
\hline 2 & 92 & 21.5 & 0.120 & 1.65 & 11 & 0.6074 & 1924 \\
\hline 2 & 34 & 22.2 & 0.118 & 1.67 & 4.0 & 0.5974 & 2970 \\
\hline 2 & 80 & 23.2 & 0.115 & 1.71 & 9.2 & 0.5841 & 3498 \\
\hline 2 & 50 & 23.6 & 0.114 & 1.73 & 5.7 & 0.5791 & 3351 \\
\hline 2 & 68 & 25.2 & 0.110 & 1.79 & 7.5 & 0.5600 & 3420 \\
\hline 2 & 92 & 30.5 & 0.100 & 1.97 & 9.2 & 0.5082 & 4234 \\
\hline 2 & 58 & 31.6 & 0.0983 & 2.00 & 5.7 & 0.4991 & 3300 \\
\hline 2 & 42 & 33.6 & 0.0953 & 2.07 & 4.0 & 0.4838 & 3680 \\
\hline 2 & 80 & 34.7 & 0.0938 & 2.10 & 7.5 & 0.4759 & 3498 \\
\hline 2 & 26 & 42.4 & 0.0847 & 2.33 & 2.2 & 0.4300 & 2720 \\
\hline 2 & 68 & 43.2 & 0.0839 & 2.35 & 5.7 & 0.4260 & 3505 \\
\hline 2 & 92 & 45.7 & 0.0816 & 2.42 & 7.5 & 0.4140 & 3848 \\
\hline 2 & 50 & 47.4 & 0.0801 & 2.46 & 4.0 & 0.4064 & 3215 \\
\hline 2 & 80 & 59.7 & 0.0713 & 2.76 & 5.7 & 0.3618 & 3505 \\
\hline 2 & 58 & 63.7 & 0.0690 & 2.86 & 4.0 & 0.3501 & 3276 \\
\hline 2 & 34 & 72.3 & 0.0647 & 3.04 & 2.2 & 0.3285 & 3549 \\
\hline 2 & 68 & 87.3 & 0.0589 & 3.35 & 4.0 & 0.2988 & 3472 \\
\hline 2 & 42 & 110 & 0.0524 & 3.76 & 2.2 & 0.2660 & 3122 \\
\hline 2 & 80 & 120 & 0.0502 & 3.93 & 4.0 & 0.02546 & 3631 \\
\hline 2 & 50 & 155 & 0.0441 & 4.47 & 2.2 & 0.2239 & 3105 \\
\hline 2 & 58 & 209 & 0.0380 & 5.19 & 2.2 & 0.1928 & 3304 \\
\hline 2 & 68 & 287 & 0.0324 & 6.08 & 2.2 & 0.1644 & 2006 \\
\hline 2 & 80 & 398 & 0.0275 & 7.16 & 2.2 & 0.1396 & 1760 \\
\hline 3 & 60 & 3.30 & 0.234 & 0.841 & 14 & 1.189 & 2752 \\
\hline 3 & 48 & 3.35 & 0.230 & 0.858 & 11 & 1.166 & 1800 \\
\hline 3 & 8 & 3.40 & 0.225 & 0.874 & 1.8 & 1.144 & 3000 \\
\hline 3 & 54 & 3.43 & 0.223 & 0.884 & 12 & 1.131 & 2295 \\
\hline 3 & 14 & 3.44 & 0.222 & 0.887 & 3.1 & 1.127 & 3600 \\
\hline 3 & 20 & 3.46 & 0.220 & 0.894 & 4.4 & 1.119 & 3160 \\
\hline 3 & 26 & 3.47 & 0.220 & 0.897 & 5.7 & 1.115 & 2840 \\
\hline 3 & 36 & 3.47 & 0.220 & 0.897 & 7.9 & 1.115 & 3300 \\
\hline 3 & 42 & 3.47 & 0.220 & 0.897 & 9.2 & 1.115 & 1725 \\
\hline 3 & 32 & 3.48 & 0.219 & 0.900 & 7.0 & 1.111 & 2996 \\
\hline 3 & 54 & 3.68 & 0.204 & 0.966 & 11 & 1.035 & 2210 \\
\hline 3 & 60 & 3.73 & 0.201 & 0.982 & 12 & 1.018 & 2752 \\
\hline 3 & 36 & 3.82 & 0.195 & 1.01 & 7.0 & 0.9883 & 3462 \\
\hline 3 & 48 & 3.86 & 0.192 & 1.03 & 9.2 & 0.9756 & 1800 \\
\hline 3 & 42 & 3.92 & 0.189 & 1.04 & 7.9 & 0.9572 & 3450 \\
\hline
\end{tabular}


Table 3 continued

\begin{tabular}{|c|c|c|c|c|c|c|c|}
\hline$d$ & $N$ & $\beta$ & $a(\mathrm{fm})$ & $a^{-1}(\mathrm{GeV})$ & $L(\mathrm{fm})$ & $m_{0}$ & Config. \\
\hline 3 & 60 & 4.01 & 0.183 & 1.07 & 11 & 0.9308 & 2752 \\
\hline 3 & 32 & 4.10 & 0.178 & 1.10 & 5.7 & 0.9058 & 3006 \\
\hline 3 & 54 & 4.25 & 0.171 & 1.15 & 9.2 & 0.8671 & 1976 \\
\hline 3 & 26 & 4.28 & 0.169 & 1.16 & 4.4 & 0.8597 & 2840 \\
\hline 3 & 42 & 4.33 & 0.167 & 1.18 & 7.0 & 0.8477 & 3277 \\
\hline 3 & 48 & 4.38 & 0.165 & 1.20 & 7.9 & 0.8360 & 1800 \\
\hline 3 & 54 & 4.83 & 0.147 & 1.34 & 7.9 & 0.7439 & 3744 \\
\hline 3 & 48 & 4.84 & 0.146 & 1.35 & 7.0 & 0.7420 & 3600 \\
\hline 3 & 36 & 4.52 & 0.159 & 1.24 & 5.7 & 0.8050 & 3300 \\
\hline 3 & 20 & 4.60 & 0.155 & 1.27 & 3.1 & 0.7883 & 3160 \\
\hline 3 & 60 & 4.64 & 0.154 & 1.28 & 9.2 & 0.7802 & 2496 \\
\hline 3 & 32 & 5.09 & 0.138 & 1.43 & 4.4 & 0.6993 & 3070 \\
\hline 3 & 42 & 5.15 & 0.136 & 1.45 & 5.7 & 0.6897 & 3400 \\
\hline 3 & 60 & 5.29 & 0.132 & 1.50 & 7.9 & 0.6685 & 4602 \\
\hline 3 & 54 & 5.36 & 0.130 & 1.52 & 7.0 & 0.6583 & 1800 \\
\hline 3 & 14 & 5.39 & 0.129 & 1.53 & 1.8 & 0.6540 & 3600 \\
\hline 3 & 36 & 5.64 & 0.122 & 1.61 & 4.4 & 0.6206 & 3300 \\
\hline 3 & 26 & 5.76 & 0.119 & 1.65 & 3.1 & 0.6057 & 2768 \\
\hline 3 & 48 & 5.78 & 0.119 & 1.66 & 5.7 & 0.6033 & 2485 \\
\hline 3 & 54 & 6.41 & 0.106 & 1.87 & 5.7 & 0.5361 & 3893 \\
\hline 3 & 42 & 6.45 & 0.105 & 1.88 & 4.4 & 0.5323 & 3450 \\
\hline 3 & 32 & 6.91 & 0.0970 & 2.03 & 3.1 & 0.4925 & 3070 \\
\hline 3 & 48 & 7.27 & 0.0917 & 2.15 & 4.4 & 0.4653 & 1800 \\
\hline 3 & 20 & 7.39 & 0.0900 & 2.19 & 1.8 & 0.4569 & 3160 \\
\hline 3 & 36 & 7.69 & 0.0861 & 2.29 & 3.1 & 0.4371 & 3387 \\
\hline 3 & 54 & 8.08 & 0.0815 & 2.42 & 4.4 & 0.4139 & 1976 \\
\hline 3 & 42 & 8.84 & 0.0739 & 2.67 & 3.1 & 0.3750 & 5359 \\
\hline 3 & 60 & 8.89 & 0.0734 & 2.68 & 4.4 & 0.3727 & 2496 \\
\hline 3 & 26 & 9.38 & 0.0692 & 2.84 & 1.8 & 0.3515 & 2840 \\
\hline 3 & 48 & 10.0 & 0.0646 & 3.05 & 3.1 & 0.3280 & 3894 \\
\hline 3 & 54 & 11.1 & 0.0577 & 3.41 & 3.1 & 0.2931 & 4973 \\
\hline 3 & 32 & 11.3 & 0.0566 & 3.48 & 1.8 & 0.2875 & 3208 \\
\hline 3 & 36 & 12.7 & 0.0500 & 3.94 & 1.8 & 0.2539 & 3410 \\
\hline 3 & 42 & 14.6 & 0.0432 & 4.57 & 1.8 & 0.2191 & 3357 \\
\hline 3 & 48 & 16.6 & 0.0377 & 5.22 & 1.8 & 0.1914 & 3769 \\
\hline 3 & 54 & 18.6 & 0.0335 & 5.88 & 1.8 & 0.1700 & 1924 \\
\hline 3 & 60 & 20.6 & 0.0301 & 6.54 & 1.8 & 0.01529 & 2400 \\
\hline 4 & 14 & 2.179 & 0.221 & 0.889 & 3.1 & 1.124 & 2930 \\
\hline 4 & 10 & 2.181 & 0.220 & 0.894 & 2.2 & 1.119 & 3000 \\
\hline 4 & 26 & 2.183 & 0.219 & 0.898 & 5.7 & 1.114 & 3152 \\
\hline 4 & 22 & 2.185 & 0.218 & 0.902 & 4.8 & 1.109 & 3186 \\
\hline 4 & 6 & 2.188 & 0.217 & 0.908 & 1.3 & 1.101 & 2220 \\
\hline 4 & 18 & 2.188 & 0.217 & 0.908 & 3.9 & 1.101 & 3284 \\
\hline 4 & 30 & 2.188 & 0.217 & 0.908 & 6.5 & 1.101 & 3000 \\
\hline 4 & 32 & 2.190 & 0.216 & 0.912 & 6.9 & 1.096 & 1646 \\
\hline 4 & 30 & 2.241 & 0.190 & 1.03 & 5.7 & 0.9667 & 3639 \\
\hline 4 & 26 & 2.252 & 0.185 & 1.06 & 4.8 & 0.9396 & 3406 \\
\hline
\end{tabular}

Table 3 continued

\begin{tabular}{llllllll}
\hline$d$ & $N$ & $\beta$ & $a(\mathrm{fm})$ & $a^{-1}(\mathrm{GeV})$ & $L(\mathrm{fm})$ & $m_{0}$ & Config. \\
\hline 4 & 22 & 2.268 & 0.177 & 1.11 & 3.9 & 0.9007 & 3096 \\
4 & 18 & 2.279 & 0.172 & 1.14 & 3.1 & 0.8743 & 3277 \\
4 & 30 & 2.305 & 0.160 & 1.23 & 4.8 & 0.8136 & 1650 \\
4 & 14 & 2.311 & 0.158 & 1.25 & 2.2 & 0.7999 & 2910 \\
4 & 26 & 2.328 & 0.150 & 1.31 & 3.9 & 0.7618 & 3256 \\
4 & 22 & 2.349 & 0.141 & 1.40 & 3.1 & 0.7162 & 2996 \\
4 & 10 & 2.376 & 0.130 & 1.52 & 1.3 & 0.6600 & 3000 \\
4 & 30 & 2.376 & 0.130 & 1.52 & 3.9 & 0.6600 & 1652 \\
4 & 18 & 2.395 & 0.123 & 1.61 & 2.2 & 0.6222 & 3105 \\
4 & 26 & 2.403 & 0.120 & 1.65 & 3.1 & 0.6067 & 3253 \\
4 & 30 & 2.448 & 0.103 & 1.91 & 3.1 & 0.5246 & 1747 \\
4 & 22 & 2.457 & 0.100 & 1.96 & 2.2 & 0.5092 & 3052 \\
4 & 14 & 2.480 & 0.0929 & 2.12 & 1.3 & 0.4714 & 2930 \\
4 & 26 & 2.507 & 0.0847 & 2.33 & 2.2 & 0.4299 & 3239 \\
4 & 30 & 2.548 & 0.0734 & 2.68 & 2.2 & 0.3726 & 1686 \\
4 & 18 & 2.552 & 0.0724 & 2.72 & 1.3 & 0.3674 & 3280 \\
4 & 22 & 2.609 & 0.0591 & 3.33 & 1.3 & 0.3001 & 3252 \\
4 & 26 & 2.656 & 0.0501 & 3.93 & 1.3 & 0.2543 & 3706 \\
4 & 30 & 2.698 & 0.0434 & 4.54 & 1.3 & 0.2204 & 1755 \\
\hline
\end{tabular}

\section{References}

1. R. Alkofer, J. Greensite, J. Phys. G 34, S3 (2007). arXiv:hep-ph/0610365

2. R. Alkofer, L. von Smekal, Phys. Rep. 353, 281 (2001). arXiv:hep-ph/0007355

3. A. Maas, Phys. Rep. 524, 203 (2013). arXiv:1106.3942

4. C.S. Fischer, J. Phys. G 32, R253 (2006). arXiv:hep-ph/0605173

5. D. Binosi, J. Papavassiliou, Phys. Rep. 479, 1 (2009). arXiv:0909.2536

6. P. Boucaud et al., Few Body Syst. 53, 387 (2012). arXiv:1109.1936

7. N. Vandersickel, D. Zwanziger, Phys. Rep. 520, 175 (2012). arXiv: 1202.1491

8. C.D. Roberts, J. Phys. Conf. Ser. 706, 022003 (2016). arXiv: 1509.02925

9. S. Strauss, C.S. Fischer, C. Kellermann, Phys. Rev. Lett. 109, 252001 (2012). arXiv:1208.6239

10. A. Cucchieri, D. Dudal, T. Mendes, N. Vandersickel, Phys. Rev. D 93, 094513 (2016). arXiv: 1602.01646

11. C.D. Roberts, A.G. Williams, Prog. Part. Nucl. Phys. 33, 477 (1994). arXiv:hep-ph/9403224

12. R. Alkofer, W. Detmold, C.S. Fischer, P. Maris, Phys. Rev. D 70, 014014 (2004). arXiv:hep-ph/0309077

13. D. August, A. Maas, JHEP 1307, 001 (2013). arXiv: 1304.4423

14. L. Fister, R. Alkofer, K. Schwenzer, Phys. Lett. B 688, 237 (2010). arXiv: 1003.1668

15. V. Macher, A. Maas, R. Alkofer, Int. J. Modern Phys. A 27, 1250098 (2012). arXiv:1106.5381

16. M. Capri et al., Ann. Phys. 343, 72 (2013). arXiv:1309.1402

17. M. Hopfer, R. Alkofer, Acta Phys. Polon. Suppl. 6, 929 (2013). arXiv: 1304.4360

18. A. Maas, PoS FacesQCD, 033 (2011). arXiv:1102.0901

19. A. Maas, T. Mufti, JHEP 1404, 006 (2014). arXiv: 1312.4873

20. A. Maas, Phys. Rev. D 75, 116004 (2007). arXiv:0704.0722 
21. A. Cucchieri, T. Mendes, Phys. Rev. Lett. 100, 241601 (2008). arXiv:0712.3517

22. A. Maas, Phys. Rev. D 91, 034502 (2015). arXiv: 1402.5050

23. H.G. Dosch, V.F. Muller, Fortschr. Phys. 27, 547 (1979)

24. A. Maas, Eur. Phys. J. C 71, 1548 (2011). arXiv:1007.0729

25. A. Cucchieri, A. Maas, T. Mendes, Phys. Rev. D 74, 014503 (2006). arXiv:hep-lat/0605011

26. J. Greensite et al., Phys. Rev. D 74, 094507 (2006). arXiv:hep-lat/0606008

27. M. Böhm, A. Denner, H. Joos, Gauge Theories of the Strong and Electroweak Interaction (Teubner, Stuttgart, 2001)

28. R. Oehme, W. Zimmermann, Phys. Rev. D 21, 471 (1980)
29. E. Seiler, Gauge Theories as a Problem of Constructive Quantum Field Theory and Statistical Mechanics (Lect. Notes Phys., 1982)

30. M. Lüscher, Commun. Math. Phys. 104, 177 (1986)

31. M. Lüscher, Commun. Math. Phys. 105, 153 (1986)

32. J. Fröhlich, G. Morchio, F. Strocchi, Phys. Lett. B 97, 249 (1980)

33. J. Fröhlich, G. Morchio, F. Strocchi, Nucl. Phys. B 190, 553 (1981)

34. A. Maas, Modern Phys. Lett. A 28, 1350103 (2013). arXiv: 1205.6625

35. M. Capri et al., Phys. Rev. D 88, 085022 (2013). arXiv:1212.1003

36. A. Maas, in preparation

37. R. Brun, F. Rademakers, Nucl. Instrum. Methods A389, 81 (1997) 